\title{
Fermionic Entanglement in Superconducting Systems
}

\author{
M. Di Tullio, ${ }^{1}$ N. Gigena, ${ }^{1}$ and R. Rossignoli ${ }^{1,2}$ \\ ${ }^{1}$ IFLP/CONICET and Departamento de Física, Universidad Nacional de La Plata, C.C. 67, La Plata (1900), Argentina \\ ${ }^{2}$ Comisión de Investigaciones Científicas (CIC), La Plata (1900), Argentina
}

\begin{abstract}
We examine distinct measures of fermionic entanglement in the exact ground state of a finite superconducting system. It is first shown that global measures such as the one-body entanglement entropy, which represents the minimum relative entropy between the exact ground state and the set of fermionic gaussian states, exhibit a close correlation with the BCS gap, saturating in the strong superconducting regime. The same behavior is displayed by the bipartite entanglement between the set of all single particle states $k$ of positive quasimomenta and their time reversed partners $\bar{k}$. In contrast, the entanglement associated with the reduced density matrix of four single particle modes $k, \bar{k}, k^{\prime}, \bar{k}^{\prime}$, which can be measured through a properly defined fermionic concurrence, exhibits a different behavior, showing a peak in the vicinity of the superconducting transition for states $k, k^{\prime}$ close to the fermi level and becoming small in the strong coupling regime. In the latter such reduced state exhibits, instead, a finite mutual information and quantum discord. And while the first measures can be correctly estimated with the BCS approximation, the previous four-level concurrence lies strictly beyond the latter, requiring at least a particle number projected BCS treatment for its description. Formal properties of all previous entanglement measures are as well discussed.
\end{abstract}

\section{INTRODUCTION}

Quantum entanglement is an essential feature of quantum mechanics. The basic notion was developed for systems of distinguishable components [1-4], where it has been extensively investigated [5 7], playing a key role in fundamental quantum information processing tasks [8, 9$]$. On the other hand, the theory of entanglement for systems of indistinguishable components such as fermions, is more recent [10 32], and is subject to some debate 33]. There are essentially two distinct approaches. One is based on the entanglement between modes $[16$ 20, 31]. Here the subsystems are defined in terms of subsets of single particle modes, and entanglement depends obviously on the choice of basis for the single particle state space and then on the choice of modes for each subsystem. The other, known as entanglement between particles $10-15,21-26]$ considers the indistinguishable constituents as subsystems and entanglement is defined beyond antisymmetrization, such that a Slater determinant is not entangled.

In 27] we defined a one-body entanglement entropy for fermion systems, which for pure states is determined by the one-body density matrix and vanishes if and only if (iff) the state is a Slater determinant. It can be obtained from a single particle mode entanglement measure after optimization over all possible bases of the single particle space. The approach can be directly extended to deal with states with no fixed fermion number (though still having fixed number parity [34]), in which case it vanishes iff the state is a quasiparticle vacuum. In the case of a single particle space of dimension 4 , the approach is an extension of the entanglement measure developed in [10] for pure states with fixed fermion number, and provides a lower bound to the entanglement associated with any bipartition of the single particle space [31]. In such space its convex roof extension can also be analytically evaluated for any mixed state in terms of the fermionic concurrence [10, 27].

The aim of this work is to analyze the previous general measures of fermionic entanglement in the exact ground state of a finite superconducting system. Previous studies of entanglement in such systems focused mainly on the formal properties of pairing correlations 15] or on the Bardeen-Cooper-Schrieffer (BCS) state [20, 35 37], using in this case methods and measures specifically devised for such state. Here we first show that the one-body entanglement entropy is in the present system a direct indicator of pairing correlations, reflecting essentially the BCS gap and saturating in the strong superconducting regime. It is also shown to be closely related to the bipartite mode entanglement between the $\Omega$ states $k$ and their time-reversed partners, becoming strictly proportional to it at the BCS level. On the other hand, the fermionic entanglement associated with four single particle modes $k \bar{k}$, $k^{\prime} \bar{k}^{\prime}$, exhibits a different behavior. This quantity is determined by a mixed reduced state with no fixed fermion number yet fixed (even) number parity, and can be explicitly evaluated through the fermionic concurrence defined in [27]. It shows a peak in the vicinity of the ground state superconducting transition for levels $k, k^{\prime}$ close to the Fermi level, becoming then small in the strong superconducting regime (if the system size is not too small). In the latter, this reduced state exhibits instead classical and discord-type 38 41] correlations, leading to a finite mutual information and quantum discord. We also discuss the BCS description of these quantities, showing that it can indeed provide a correct estimation of the first measures in the superconducting phase, although it fails to describe the four-mode fermionic concurrence, which is identically zero in BCS for all coupling strengths. This quantity is shown to require at least a projected BCS treatment.

In sec.【we first discuss the main properties of the em- 
ployed fermionic entanglement measures, some of them not included in [27, 31], showing in particular their direct relation with the minimum relative entropy to a fermionic gaussian state. It is also shown that in the case of four single particle modes, their extension to mixed states also warrants, if non-zero, a finite bipartite mode entanglement for any partition of the single particle space. The application of these measures to the exact ground state of a finite superconducting system is discussed in sec. III A where their behavior as a function of the pairing coupling strength is analyzed. Their description through the BCS approximation is discussed in IIIB which also includes a simple projected (before variation) BCS treatment, necessary for describing the four-mode fermionic concurrence. Other quantities like the mutual information and quantum discord of four single particle modes, are also discussed in III and in the Appendices, which contain additional details. Exact analytic expressions for the strong coupling regime are as well provided.

\section{FORMALISM}

\section{A. One-body entanglement entropies}

We consider a fermion system described by a single particle space $\mathcal{H}$, spanned by fermion operators $c_{i}, c_{i}^{\dagger}$, $i=1, \ldots, d$, satisfying the anticommutation relations $\left\{c_{i}, c_{j}^{\dagger}\right\}=\delta_{i j},\left\{c_{i}, c_{j}\right\}=\left\{c_{i}^{\dagger}, c_{j}^{\dagger}\right\}=0$. Given a pure state $|\Psi\rangle$ of this system, the set of averages

$$
\rho_{i j}^{\mathrm{sp}}=\left\langle c_{j}^{\dagger} c_{i}\right\rangle \equiv\left\langle\Psi\left|c_{j}^{\dagger} c_{i}\right| \Psi\right\rangle,
$$

form the one-body density matrix $\rho^{\mathrm{sp}}=\mathbb{1}-\left\langle\boldsymbol{c} \boldsymbol{c}^{\dagger}\right\rangle(\boldsymbol{c}=$ $\left.\left(c_{1}, \ldots, c_{d}\right)^{t}\right)$. It plays the role of a "reduced" density matrix which determines the average of any one-body operator $\hat{O}=c^{\dagger} O c=\sum_{i, j} O_{i j} c_{i}^{\dagger} c_{j}:\langle\hat{O}\rangle=\operatorname{tr}\left[\rho^{\mathrm{sp}} O\right]$, with $\operatorname{tr}$ denoting the trace in the single particle space.

In [27] we have defined an associated one-body entanglement entropy,

$$
\begin{aligned}
E(|\Psi\rangle) & =\operatorname{tr}\left[h\left(\rho^{\mathrm{sp}}\right)\right]=\sum_{i} h\left(f_{i}\right), \\
h\left(f_{i}\right) & =-f_{i} \log _{2} f_{i}-\left(1-f_{i}\right) \log _{2}\left(1-f_{i}\right),
\end{aligned}
$$

where $f_{i}=\left\langle a_{i}^{\dagger} a_{i}\right\rangle$ are the eigenvalues of $\rho^{\mathrm{sp}}$ and $\boldsymbol{a}=U^{\dagger} \boldsymbol{c}$ is the set of fermion operators diagonalizing $\rho^{\mathrm{sp}}$, such that $\left\langle a_{j}^{\dagger} a_{i}\right\rangle=\left(U^{\dagger} \rho^{\mathrm{sp}} U\right)_{i j}=f_{i} \delta_{i j}$, with $U^{\dagger} U=\mathbb{1}$.

Eq. (2) vanishes iff $f_{i}=0$ or $1 \forall i$, i.e., iff $|\Psi\rangle$ is a Slater determinant $\left(|\Psi\rangle=\left[\prod_{i}\left(a_{i}^{\dagger}\right)^{f_{i}}\right]|0\rangle\right)$, and remains obviously invariant under one-body unitary transformations $|\Psi\rangle \rightarrow \exp \left[-i \boldsymbol{c}^{\dagger} O \boldsymbol{c}\right]|\Psi\rangle\left(O^{\dagger}=O\right)$, which just lead to a unitary transformation of $\rho^{\mathrm{sp}}\left(\rho^{\mathrm{sp}} \rightarrow e^{-i O} \rho^{\mathrm{sp}} e^{i O}\right)$. It is also the minimum, over all single particle bases, of the entropy determined by the average occupation of the corresponding single particle states [27]:

$$
E(|\Psi\rangle)=\underset{\left\{c_{i}\right\}}{\operatorname{Min}} \sum_{i} h\left(\left\langle c_{i}^{\dagger} c_{i}\right\rangle\right),
$$

with $h\left(\left\langle c_{i}^{\dagger} c_{i}\right\rangle\right)$ representing the entanglement entropy of a single particle mode with the remaining modes (see also sec. (IIB).

Eq. (2) also admits other interpretations. It can be regarded as the von Neumann entropy $S\left(\rho^{\prime}\right)$, in the grand canonical ensemble, of the independent fermion density operator $\rho^{\prime}$ which reproduces the whole single particle density matrix determined by $|\Psi\rangle$ : If

$$
\rho^{\prime}=Z^{-1} \exp \left[-\boldsymbol{c}^{\dagger} \Lambda \boldsymbol{c}\right]=Z^{-1} \exp \left[-\sum_{i} \lambda_{i} a_{i}^{\dagger} a_{i}\right],
$$

with $Z=\operatorname{Tr} \exp \left[-\boldsymbol{c}^{\dagger} \Lambda \boldsymbol{c}\right]=\prod_{i}\left(1+e^{-\lambda_{i}}\right)$ and $\lambda_{i}$ the eigenvalues of the matrix $\Lambda$, then

$$
S\left(\rho^{\prime}\right)=-\operatorname{Tr}\left[\rho^{\prime} \log _{2} \rho^{\prime}\right]=\sum_{i} h\left(f_{i}\right),
$$

where $f_{i}=\operatorname{Tr}\left[\rho^{\prime} a_{i}^{\dagger} a_{i}\right]=\left[1+e^{\lambda_{i}}\right]^{-1}$. Eq. (6) will then coincide with (2) provided these $f_{i}$ 's are identical with the eigenvalues of the single particle density matrix (1), i.e., provided

$$
\mathbb{1}-\operatorname{tr}\left[\rho^{\prime} \boldsymbol{c} \boldsymbol{c}^{\dagger}\right]=[\mathbb{1}+\exp (\Lambda)]^{-1}=\rho^{\mathrm{sp}},
$$

which implies $\Lambda=\ln \left[\left(\rho^{\mathrm{sp}}\right)^{-1}-\mathbb{1}\right]$.

This result shows that Eq. (2) is in fact the relative entropy [43, 44] (in the grand canonical ensemble) between the pure state $\rho=|\Psi\rangle\langle\Psi|$ and the state (5) which satisfies (7), since $S(\rho)=0$ and (7) implies $\operatorname{Tr}\left[\rho \log _{2} \rho^{\prime}\right]=$ $\operatorname{Tr}\left[\rho^{\prime} \log _{2} \rho^{\prime}\right]$ :

$$
\begin{aligned}
S\left(\rho \| \rho^{\prime}\right) & \equiv-\operatorname{Tr}\left[\rho\left(\log _{2} \rho^{\prime}-\log _{2} \rho\right)\right] \\
& =S\left(\rho^{\prime}\right)=\operatorname{tr}\left[h\left(\rho^{\mathrm{sp}}\right)\right] .
\end{aligned}
$$

Moreover, as shown in Appendix A Eq. (9) is also the minimum relative entropy (in the grand canonical ensemble) between $\rho$ and any operator of the form (5):

$$
\operatorname{Min}_{\rho^{\prime}} S\left(\rho \| \rho^{\prime}\right)=\operatorname{tr}\left[h\left(\rho^{\mathrm{sp}}\right)\right] .
$$

Hence, Eq. (2) is a measure of the minimum distance between $\rho$ and the set of operators of the form (5) (fermionic gaussian states commuting with $N$ ).

Extension to quasiparticles. If the state $|\Psi\rangle$ does not have a fixed fermion number $N=\sum_{i} c_{i}^{\dagger} c_{i}$, (but has a definite number parity $e^{i \pi N}= \pm 1$, in agreement with the parity superselection rule [34]), we can define a generalized one-body entanglement entropy [27] based on the extended one-body density matrix $\rho^{\mathrm{qsp}}$, which contains the contractions $\kappa_{i j}=\left\langle c_{j} c_{i}\right\rangle$ and $-\kappa_{i j}^{*}=\left\langle c_{j}^{\dagger} c_{i}^{\dagger}\right\rangle$ :

$$
\begin{aligned}
& E^{\mathrm{qsp}}(|\Psi\rangle)=-\operatorname{tr}\left[\rho^{\mathrm{qsp}} \log _{2} \rho^{\mathrm{qsp}}\right]=\sum_{i} h\left(\tilde{f}_{i}\right), \\
& \rho^{\mathrm{qsp}}=\mathbb{1}-\left\langle\left(\begin{array}{c}
\boldsymbol{c} \\
\boldsymbol{c}^{\dagger t}
\end{array}\right)\left(\begin{array}{ll}
\boldsymbol{c}^{\dagger} & \boldsymbol{c}^{t}
\end{array}\right)\right\rangle=\left(\begin{array}{cc}
\rho^{\mathrm{sp}} & \kappa \\
-\kappa^{*} & \mathbb{1}-\left(\rho^{\mathrm{sp}}\right)^{*}
\end{array}\right)
\end{aligned}
$$

Here $\tilde{f}_{i}=\left\langle\tilde{a}_{i}^{\dagger} \tilde{a}_{i}\right\rangle$ and $1-\tilde{f}_{i}=\left\langle\tilde{a}_{i} \tilde{a}_{i}^{\dagger}\right\rangle$ are the eigenvalues of $\rho^{\mathrm{qsp}}$ (which always come in pairs $\left(\tilde{f}_{i}, 1-\tilde{f}_{i}\right)$ ), with $\tilde{a}_{i}$ 
the fermion quasiparticle operators diagonalizing $\rho^{\mathrm{qsp}}$, related to the original operators $c_{i}, c_{i}^{\dagger}$ through a Bogoliubov transformation [45]. Eq. (11) reduces to (2) iff $\kappa=0$, and vanishes iff $|\Psi\rangle$ is a Slater determinant or also a quasiparticle vacuum (or equivalently, a quasiparticle Slater determinant, which can be always written as a quasiparticle vacuum through a particle-hole transformation). Eq. (11) remains invariant under arbitrary particle hole transformations $\left(c_{i} \rightarrow c_{i}^{\dagger}\right.$ for some single particle states $i$ ), as well as arbitrary Bogoliubov transformations [27]. It is the minimum, over all single quasiparticle bases, of the sum of the entanglement entropies of all single quasiparticle modes [27].

Eq. (11) is also the minimum relative entropy between $\rho$ and any fermionic gaussian state, i.e.. any $\rho^{\prime}$ which is the exponent of a generalized one-body operator:

$$
\begin{gathered}
\operatorname{Min}_{\rho^{\prime}} S\left(\rho \| \rho^{\prime}\right)=-\operatorname{Tr}\left[\rho^{\mathrm{qsp}} \log _{2} \rho^{\mathrm{qsp}}\right], \\
\rho^{\prime}=Z^{-1} \exp \left[-\boldsymbol{c}^{\dagger} \Lambda \boldsymbol{c}-\frac{1}{2}\left(\boldsymbol{c}^{\dagger} \Gamma \boldsymbol{c}^{\dagger t}+\boldsymbol{c}^{t} \Gamma^{\dagger} \boldsymbol{c}\right)\right] \\
=\tilde{Z}^{-1} \exp \left[-\left(\boldsymbol{c}^{\dagger} \boldsymbol{c}^{t}\right) \mathcal{L}\left(\begin{array}{c}
\boldsymbol{c} \\
\boldsymbol{c}^{\dagger t}
\end{array}\right)\right], \mathcal{L}=\left(\begin{array}{cc}
\Lambda & \Gamma \\
-\Gamma^{*} & \mathbb{1}-\Lambda^{*}
\end{array}\right) .
\end{gathered}
$$

The minimum (13) is reached for that $\rho^{\prime}$ which reproduces the full $\rho^{\mathrm{qsp}}$, i.e., that satisfying

$$
\mathbb{1}-\operatorname{tr}\left[\rho^{\prime}\left(\begin{array}{c}
\boldsymbol{c} \\
\boldsymbol{c}^{\dagger t}
\end{array}\right)\left(\boldsymbol{c}^{\dagger} \boldsymbol{c}^{t}\right)\right]=[1+\exp (\mathcal{L})]^{-1}=\rho^{\mathrm{qsp}},
$$

which implies $\mathcal{L}=\ln \left[\left(\rho^{\mathrm{qsp}}\right)^{-1}-1\right]$ and hence $S\left(\rho^{\prime}\right)=$ $-\operatorname{tr}\left[\rho^{\mathrm{qsp}} \log _{2} \rho^{\mathrm{qsp}}\right]$.

\section{B. Entanglement of bipartitions of the single particle space}

Given a decomposition $\mathcal{H}_{A} \oplus \mathcal{H}_{B}$ of the single particle space $\mathcal{H}$ in orthogonal subspaces of finite dimension $d_{A}, d_{B}=d-d_{A}$, we may expand $|\Psi\rangle$ in a set of Slater determinant in $\mathcal{H}_{A}$ and $\mathcal{H}_{B}$ as $|\Psi\rangle=\sum_{\mu, \nu} \alpha_{\mu \nu}|\mu \nu\rangle$, where $|\mu \nu\rangle=\left[\prod_{i \in A}\left(c_{i}^{\dagger}\right)^{n_{i}^{\nu}}\right]\left[\prod_{j \in B}\left(c_{j}^{\dagger}\right)^{n_{j}^{\mu}}\right]|0\rangle$, with $n_{i}^{\nu}=$ 0,1 the occupation number of single particle state $i$ in Slater determinant $\nu$. The reduced states associated with these single particle subspaces are [29] $\rho_{A}=$ $\sum_{\mu, \mu^{\prime}}\left(\alpha \alpha^{\dagger}\right)_{\mu \mu^{\prime}}|\mu\rangle\left\langle\mu^{\prime}\right|$ and $\rho_{B}=\sum_{\nu, \nu^{\prime}}\left(\alpha^{t} \alpha^{*}\right)_{\nu \nu^{\prime}}|\nu\rangle\left\langle\nu^{\prime}\right|$, which reproduce all expectation values of operators containing creation and annihilation operators acting just on these subspaces. They are normalized mixed states with the same non-zero eigenvalues $\lambda_{k}$, given by the singular values of the matrix $\alpha$. Their entropies $S\left(\rho_{A}\right)=$ $S\left(\rho_{B}\right)=-\sum_{k} \lambda_{k} \log _{2} \lambda_{k}$ represent the entanglement entropy $E(A, B)$ associated with this partition [27, 29].

For states $|\Psi\rangle$ having definite fermion number, $\rho_{A(B)}$ will commute with the local fermion number $N_{A(B)}=$ $\sum_{i \in A(B)} c_{i}^{\dagger} c_{i}$, but will in general be a mixture of states with different particle number (it will be represented by a $2^{d_{A(B)}} \times 2^{d_{A(B)}}$ matrix). Similarly, if $|\Psi\rangle$ has definite number parity, $\rho_{A(B)}$ will commute with the local number parity $e^{i \pi N_{A(B)}}$, being a mixture of even and odd states.

For instance, if $\mathcal{H}_{A}$ involves just one single particle level $i, \rho_{A} \equiv \rho_{i}$ will be determined by the average occupation $\left\langle c_{i}^{\dagger} c_{i}\right\rangle$ :

$$
\rho_{i}=\left(\begin{array}{cc}
\left\langle c_{i}^{\dagger} c_{i}\right\rangle & 0 \\
0 & \left\langle c_{i} c_{i}^{\dagger}\right\rangle
\end{array}\right)
$$

in the basis $\left\{c_{i}^{\dagger}|0\rangle,|0\rangle\right\}$, with $\left\langle c_{i} c_{i}^{\dagger}\right\rangle=1-\left\langle c_{i} c_{i}^{\dagger}\right\rangle\left(\left\langle c_{i}\right\rangle=0\right.$ due to number parity conservation). Its entropy $S\left(\rho_{i}\right)=$ $h\left(\left\langle c_{i}^{\dagger} c_{i}\right\rangle\right)$ represents the entanglement entropy of such mode with the remaining modes. A single particle basis where $S\left(\rho_{i}\right)=0 \forall i$ exists iff Eq. (2) vanishes. And a single quasiparticle basis with the same property exists iff $E^{\mathrm{qsp}}(|\Psi\rangle)=0$.

Similarly, if $\mathcal{H}_{A}$ comprises a pair of levels $i \neq j$, then

$$
\rho_{i j}=\left(\begin{array}{cccc}
\left\langle c_{i}^{\dagger} c_{i} c_{j}^{\dagger} c_{j}\right\rangle & 0 & 0 & \left\langle c_{j} c_{i}\right\rangle \\
0 & \left\langle c_{i}^{\dagger} c_{i} c_{j} c_{j}^{\dagger}\right\rangle & \left\langle c_{j}^{\dagger} c_{i}\right\rangle & 0 \\
0 & \left\langle c_{i}^{\dagger} c_{j}\right\rangle & \left\langle c_{i} c_{i}^{\dagger} c_{j}^{\dagger} c_{j}\right\rangle & 0 \\
\left\langle c_{i}^{\dagger} c_{j}^{\dagger}\right\rangle & 0 & 0 & \left\langle c_{i} c_{i}^{\dagger} c_{j} c_{j}^{\dagger}\right\rangle
\end{array}\right),
$$

in the basis $\left\{c_{i}^{\dagger} c_{j}^{\dagger}|0\rangle, c_{i}^{\dagger}|0\rangle, c_{j}^{\dagger}|0\rangle,|0\rangle\right\}$. This reduced state $\left(\operatorname{Tr} \rho_{i j}=1\right)$ determines the average of any operator involving just $c_{i}, c_{j}, c_{i}^{\dagger}, c_{j}^{\dagger}$, and its entropy $S\left(\rho_{i j}\right)$ is the entanglement entropy of this pair of single particle modes with the remaining modes. The outer (inner) block of $\rho_{i j}$ corresponds to positive (negative) pair number parity. In contrast with $\rho_{i}, \rho_{i j}$ is not fully determined by $\rho^{\mathrm{qsp}}$ since its diagonal elements (i.e. the probabilities of finding none, one or both levels occupied) involve twobody contractions. Nonetheless, if $c_{i}, c_{j}$ are operators diagonalizing $\rho^{\mathrm{qsp}}\left(c_{i(j)} \rightarrow \tilde{a}_{i(j)}\right), \rho_{i j}$ is obviously diagonal and $E(|\Psi\rangle)=0$ or $E^{\mathrm{qsp}}(|\Psi\rangle)=0$ implies $S\left(\rho_{i j}\right)=0$ for such operators (just one of the diagonal elements will be non-zero).

Note also that $S\left(\rho_{i j}\right)$ depends on the subspace spanned by the single particle levels $i, j$, but not on the particular choice of states $i, j$ within this subspace: Any unitary or Bogoliubov transformation involving just $c_{i}, c_{i}^{\dagger}, c_{j}, c_{j}^{\dagger}$ will leave such entropy invariant.

\section{Entanglement of subspaces of the single particle space}

Let us now consider the entanglement of a reduced state $\rho_{A}$ of a single particle subspace $\mathcal{H}_{A}$. For reduced states which commute with the local fermion number $N_{A}$, we define the associated one-body entanglement of formation as

$$
E\left(\rho_{A}\right)=\operatorname{Min}_{\sum_{\alpha} q_{\alpha}\left|\Psi_{\alpha}^{A}\right\rangle\left\langle\Psi_{\alpha}^{A}\right|=\rho_{A}} q_{\alpha} E\left(\left|\Psi_{\alpha}^{A}\right\rangle\right),
$$

where $q_{\alpha} \geq 0, \sum_{\alpha} q_{\alpha}=1$ and the minimization is over all representations of $\rho_{A}$ as a convex combination of pure 
states in $\mathcal{H}_{A}$ with definite fermion number. Eq. (17) vanishes iff $\rho_{A}$ can be written as a convex mixture of Slater Determinants. Similarly, we define the generalized one-body entanglement of formation as

$$
E^{\mathrm{qsp}}\left(\rho_{A}\right)=\operatorname{Min}_{\sum_{\alpha} q_{\alpha}\left|\Psi_{\alpha}^{A}\right\rangle\left\langle\Psi_{\alpha}^{A}\right|=\rho_{A}} q_{\alpha} E^{\mathrm{qsp}}\left(\left|\Psi_{\alpha}^{A}\right\rangle\right),
$$

where the minimization is now over all representations of $\rho_{A}$ as convex combination of pure states with definite number parity. Eq. (18) vanishes iff $\rho_{A}$ can be written as a convex mixture of quasiparticle vacua or Slater Determinants.

It is first apparent that if the full state $|\Psi\rangle$ is a quasiparticle vacuum or Slater determinant, then $E^{\mathrm{qsp}}\left(\rho_{A}\right)=$ 0 for any subspace $\mathcal{H}_{A}$ : In this case all averages involved in the construction of $\rho_{A}$ can be determined through Wick's theorem [45], and hence expressed in terms of the elements of $\rho^{\text {qsp }}$ involving this subspace. Therefore, $\rho_{A}$ can be written as the exponent of a suitable generalized one-body operator of the form (14) providing the same $\rho^{\mathrm{qsp}}$ for this subspace, and will then be a convex combination of quasiparticle Slater Determinants or vacua. A non-zero value of $E^{\mathrm{qsp}}\left(\rho_{A}\right)$ is then indicative of correlations beyond those provided by a global quasiparticle vacuum. Similarly, if $|\Psi\rangle$ is a standard Slater determinant then $E\left(\rho_{A}\right)=0$, since in this case $\kappa=0$ and $\rho_{A}$ can then be written as an operator of the form (5).

It is also apparent that for a single level $i, E\left(\rho_{i}\right)=$ $E^{\mathrm{qsp}}\left(\rho_{i}\right)=0$ for any $|\Psi\rangle$. Similarly, for two single particle levels we always have $E^{\mathrm{qsp}}\left(\rho_{i j}\right)=0$, since any pure state with fixed number parity in a two-dimensional single particle space (such as the eigenstates of $\rho_{i j}$ ) can be written as a quasiparticle vacuum or Slater determinant [27, 29]. And if $\left\langle c_{i} c_{j}\right\rangle=0$ (i.e. $\left[\rho_{i j}, N_{i j}\right]=0$ ) then $E\left(\rho_{i j}\right)=0$. The same property holds for three distinct levels $i, j, k$ for the same reason: Any pure state with fixed number parity in a three-dimensional single particle space can be written as a quasiparticle vacuum or Slater determinant [27], implying $E^{\mathrm{qsp}}\left(\rho_{i j k}\right)=0$ (and also $E\left(\rho_{i j k}\right)=0$ if $\left.\left[\rho_{i j k}, N_{i j k}\right]=0\right)$.

The first non-trivial case is that of four distinct single particle levels $i, j, k, l$, in which case a closed expression for the one-body entanglement of formation for any state $\rho_{i j k l}$ with fixed number parity was obtained in [27], extending the results of [10] for states with fixed fermion number. The result is

$$
E^{\mathrm{qsp}}\left(\rho_{i j k l}\right)=-4 \sum_{\nu= \pm} f_{\nu} \log f_{\nu}, \quad f_{ \pm}=\frac{1 \pm \sqrt{1-C^{2}\left(\rho_{i j k l}\right)}}{2}
$$

where $C$ is the corresponding fermionic concurrence $[10$, 27],

$$
C\left(\rho_{i j k l}\right)=\operatorname{Max}\left[2 \lambda_{\max }-\operatorname{Tr} R\left(\rho_{i j k l}\right), 0\right],
$$

with $\lambda_{\max }$ the largest eigenvalue of $R(\rho)=\sqrt{\rho^{1 / 2} \tilde{\rho} \rho^{1 / 2}}$ and $\tilde{\rho}=T \rho^{*} T$ in a standard basis. The operation $T$ is given explicitly in Appendix B (note that $\rho_{i j k l}$ and $T$ are represented by $8 \times 8$ matrices).
For a pure $\rho_{i j k l}=|\Psi\rangle\langle\Psi|, C^{2}$ becomes a quadratic entropy of the corresponding four-level $\rho^{\mathrm{qsp}}: C^{2}(|\Psi\rangle)=$ $\frac{1}{2} \operatorname{Tr} \rho^{\mathrm{qsp}}\left(1-\rho^{\mathrm{qsp}}\right)=4 f_{+} f_{-}$, with $f_{+}, f_{-}=1-f_{+}$the (four-fold degenerate) eigenvalues of $\rho^{\mathrm{qsp}}$ [27]. For a mixed $\rho_{i j k l}$, the result (20) coincides with the convex roof extension of $C(|\Psi\rangle)$ [Eq. (18) with $E^{\mathrm{qsp}}\left(\left|\Psi_{\alpha}^{A}\right\rangle\right)$ replaced by $\left.C\left(\left|\Psi_{\alpha}^{A}\right\rangle\right)\right]$. If $\rho_{i j k l}$ commutes with number parity but contains components of both parities, it can be written as a mixture $\sum_{p= \pm} q_{p} \rho_{i j k l}^{p}$ of two mixed states with definite number parity and $E^{\mathrm{qsp}}\left(\rho_{i j k l}\right)$ can be evaluated as the average of the expressions for each parity [27].

We can also consider bipartitions $\left(A_{1}, A_{2}\right)$ of the $\mathcal{H}_{A}$ subspace, with $\mathcal{A}_{1}$ and $\mathcal{A}_{2}$ of nonzero dimension and determined by a given choice of levels in some single particle basis of $\mathcal{H}_{A}$, and examine the associated bipartite entanglement in the state $\rho_{A_{1}, A_{2}}$. Such state will be separable iff it can be written as a convex combination of pure product states (with definite number parity) in $A_{1}$ and $A_{2}$, and entangled otherwise. While there is in general no relation between this entanglement and $E^{\mathrm{qsp}}\left(\rho_{A}\right)$, in the case of four single particle levels, it was shown in [31] that for a pure $\rho_{i j k l}=|\Psi\rangle\langle\Psi|$, the quantity $\frac{1}{4} E^{\mathrm{qsp}}(|\Psi\rangle)$ provides a lower bound to the entanglement entropy of any bipartition of the single particle space.

For a general $\rho_{i j k l}$, we now show the following Lemma, which relates the fermionic concurrence (19) with the entanglement of a bipartition: For a general four-level fermionic state $\rho_{i j k l}$ commuting with number parity and satisfying $C\left(\rho_{i j k l}\right)>0$, any bipartition of $\mathcal{H}_{i j k l}$ (like $(i j-k l)$ or $i-j k l)$ is entangled.

Proof: If $\rho_{i j k l}$ were separable for a given bipartition $A_{1}-A_{2}$, it could be written as a convex combination of pure product states $\left|\mu_{A_{1}}\right\rangle\left\langle\mu_{A_{1}}|\otimes| \nu_{A_{2}}\right\rangle\left\langle\nu_{A_{2}}\right|$, with $\left|\mu_{A_{1}}\right\rangle$, $\left|\nu_{A_{2}}\right\rangle$ having definite number parity. But since they are definite number parity pure states in a single particle space of dimension $d \leq 3$, they are necessarily a Slater determinant or quasiparticle vacuum, as discussed above. Consequently, $\rho_{i j k l}$ can be written as a convex combination of Slater determinant or quasiparticle vacua $\left|\mu_{A 1} \nu_{A 2}\right\rangle\left\langle\mu_{A_{1}} \nu_{A 2}\right|$, of definite number parity, entailing $C\left(\rho_{i j k l}\right)=0$. Thus, $C\left(\rho_{i j k l}\right)>0$ ensures that any bipartition of $\mathcal{H}_{A}$ is entangled, for any choice of single particle or quasiparticle basis of this subspace.

\section{APPLICATION TO A FINITE PAIRING SYSTEM}

\section{A. Exact results}

We now consider a fermion system with a single particle space $\mathcal{H}$ of finite dimension $2 \Omega$, spanned by $\Omega$ orthogonal single particle states $k$ and the corresponding $\Omega$ time-reversed states $\bar{k}$. We consider in such space a pairing Hamiltonian of the form

$$
H=\sum_{k} \varepsilon_{k}\left(c_{k}^{\dagger} c_{k}+c_{\bar{k}}^{\dagger} c_{\bar{k}}\right)-\sum_{k, k^{\prime}} G_{k k^{\prime}} c_{k^{\prime}}^{\dagger} c_{\bar{k}^{\prime}}^{\dagger} c_{\bar{k}} c_{k}
$$


where $\varepsilon_{k}$ are the single particle energies (the same for $k$ and $\bar{k}$ states) and the pairing interaction moves pairs of fermions from $k, \bar{k}$ to $k^{\prime}, \bar{k}^{\prime}$. We will focus on an equally spaced single particle spectrum $\varepsilon_{k+1}-\varepsilon_{k}=\varepsilon \forall k$, with a constant coupling strength $G_{k k^{\prime}}=G \geq 0 \forall k, k^{\prime}$, and examine the half-filled case where the number of fermions is $N=\Omega$, with $\Omega$ even.

The exact ground state will then be a linear combination of Slater Determinants with fixed fermion number $N$ and fully occupied or empty pairs $(k, \bar{k})$ :

$$
|\Psi\rangle=\sum_{\nu} \alpha_{\nu}\left[\prod_{k}\left(c_{k}^{\dagger} c_{\frac{k}{k}}^{\dagger}\right)^{n_{k}^{\nu}}\right]|0\rangle
$$

where $n_{k}^{\nu}=0,1$ indicates the occupation of pair $k, \bar{k}$ $\left(\sum_{k} n_{k}^{\nu}=N / 2\right)$ and $\nu=1, \ldots,\left(\begin{array}{c}\Omega \\ N / 2\end{array}\right)$ runs over these Slater Determinants $\left(\sum_{\nu}\left|\alpha_{\nu}^{2}\right|=1\right)$. This state leads to a single particle density matrix which remains strictly diagonal in the unperturbed single particle basis: $\left\langle c_{k}^{\dagger} c_{\bar{k}^{\prime}}\right\rangle=$ $0 \forall k, k^{\prime}$ and

$$
\left\langle c_{k}^{\dagger} c_{k^{\prime}}\right\rangle=\left\langle c_{\bar{k}}^{\dagger} c_{\bar{k}^{\prime}}\right\rangle=\delta_{k k^{\prime}} f_{k},
$$

where $f_{k}=\left\langle c_{k}^{\dagger} c_{k}\right\rangle=\left\langle c_{\bar{k}}^{\dagger} c_{\bar{k}}\right\rangle=\sum_{\nu}\left|\alpha_{\nu}^{2}\right| n_{k}^{\nu}$ is the average occupation of single particle state $k$ or $\bar{k}$ in the exact ground state (22) $\left(2 \sum_{k} f_{k}=N\right)$. Since no off-diagonal terms arise, these $f_{k}$ are the eigenvalues of $\rho^{\mathrm{sp}}$.

\section{One-body entanglement entropy and global bipartite entanglement}

The exact one-body entanglement entropy (2) then becomes

$$
E(|\Psi\rangle)=2 \sum_{k} h\left(f_{k}\right)
$$

where $h\left(f_{k}\right)=-f_{k} \log _{2} f_{k}-\left(1-f_{k}\right) \log _{2}\left(1-f_{k}\right)$ represents the entropy $S\left(\rho_{k}\right)$ of the single mode density [Eq. (15)]

$$
\rho_{k}=\rho_{\bar{k}}=\left(\begin{array}{cc}
f_{k} & 0 \\
0 & 1-f_{k}
\end{array}\right),
$$

i.e., the single mode entropy. We remark that for the exact ground state, $E(|\Psi\rangle)=E^{\mathrm{qsp}}(|\Psi\rangle)$ since $|\Psi\rangle$ has a fixed $N$. We also note that $h\left(f_{k}\right)$ is an increasing function of the occupation number fluctuation

$$
\left\langle n_{k}^{2}\right\rangle-\left\langle n_{k}\right\rangle^{2}=f_{k}\left(1-f_{k}\right)=\frac{1}{4} S_{2}\left(\rho_{k}\right),
$$

where $S_{2}(\rho)=2 \operatorname{Tr}[\rho(\mathbb{1}-\rho)]$ is the quadratic (also called linear) entropy. Eq. (26) is then also a measure of single mode entanglement. The relation between entanglement and fluctuations (and also higher order cumulants) has been discussed in detail in [47].

A plot of (24) for the exact ground state (obtained by diagonalization of $H$ ) of a system with $2 \Omega=32$ single

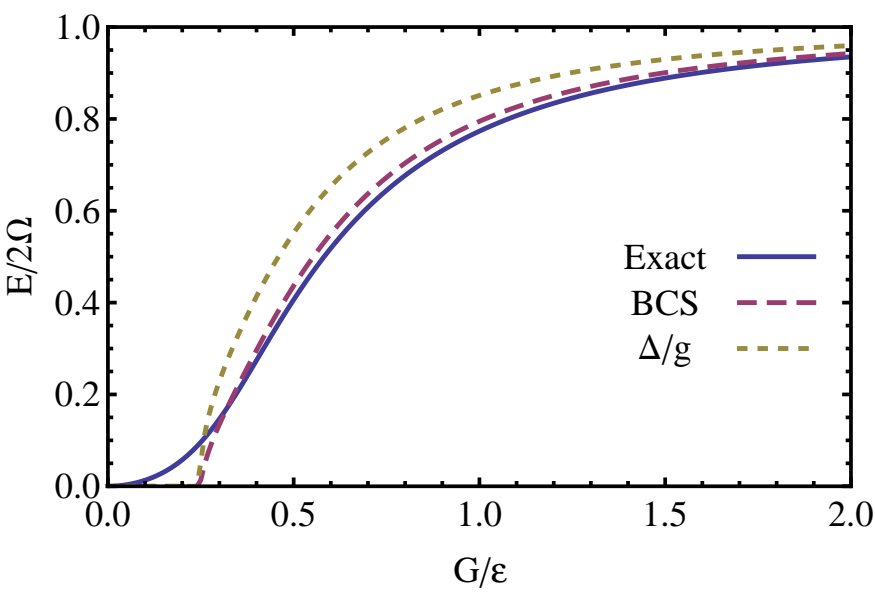

FIG. 1. Intensive one body entanglement entropy $E(|\Psi\rangle) /(2 \Omega)$ (Eq. (24), dimensionless), in the ground state of the Hamiltonian (21) as a function of the (dimensionless) scaled coupling strength $G / \varepsilon$ ( $\varepsilon$ is the single particle level spacing) for $2 \Omega=32$ single particle levels and $N=\Omega$. Exact and BCS results are depicted. The scaled BCS gap $\Delta / g$, with $g=G \Omega / 2$, is also shown. All quantities approach 1 for $G /(\Omega \varepsilon) \rightarrow \infty$. BCS results vanish for $G<G_{c}$. Quantities plotted are dimensionless in all figures.

particle states is depicted in Fig. 1, This entropy, which measures the deviation of (22) from a Slater determinant, is seen here to be a direct indicator of pairing correlations, becoming nonzero for all $G>0$ and large in the BCS superconducting phase $G>G_{c}$ (see IIIB). Its behavior for $G>G_{c}$ resembles in fact that of the scaled BCS gap $\Delta / G$ (also depicted). Pairing correlations smooth out the original Fermi surface, leading to finite average occupations $0<f_{k}<1 / 2$ for single particle levels above the Fermi level, which increase with increasing $G$, and $1 / 2<f_{k}<1$ for levels below the Fermi level, which decrease with increasing $G$. Then each term $h\left(f_{k}\right)$ in the sum (24) increases as $G$ increases, giving rise to the previous behavior of $E(|\Psi\rangle)$. While for $G>G_{c}$ these effects can be correctly described with the BCS approach, in a finite system pairing effects in the exact ground state become also visible within the weak coupling sector $0<$ $G<G_{c}$, where BCS results vanish. For any $G>0$ and finite $\Omega$, the coupling will mix all states in the expansion (22), leading to $\alpha_{\nu}>0 \forall \nu$ and hence to $0<f_{k}<1$ $\forall k$. The state (22) will then cease to be a strict Slater determinant as soon as $G$ increases from 0 (see also end of Appendix (C).

As seen in Fig. 2, the increase with $G$ of the single mode entropies $h\left(f_{k}\right)$ will obviously be initially more rapid for levels close to the Fermi level, since their occupation will be more strongly affected by the coupling. The occupation number fluctuation $f_{k}\left(1-f_{k}\right)$ rapidly increases for these levels, leading to a larger $h\left(f_{k}\right)$. The finite value of $E(|\Psi\rangle)$ for $0<G<G_{c}$ is precisely due to the non-negligible contributions $h\left(f_{k}\right)$ from levels close to the 


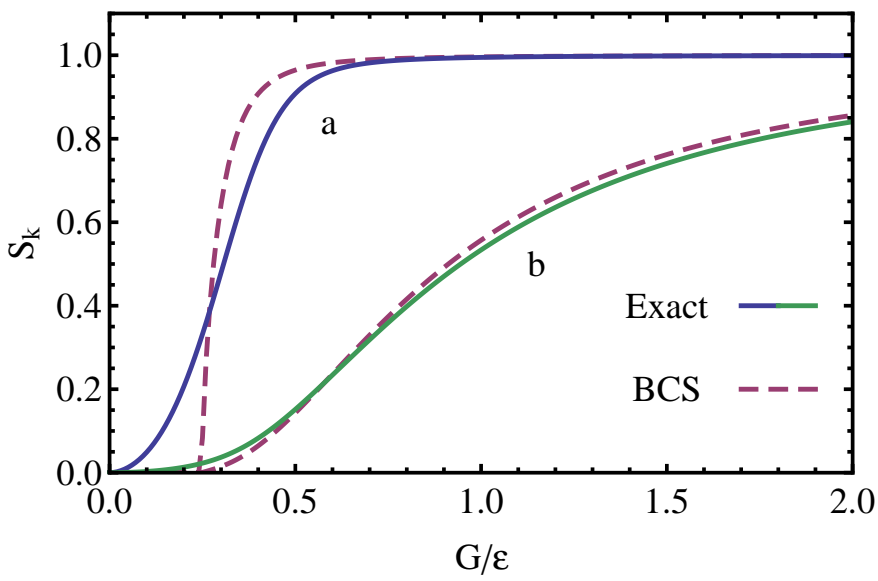

FIG. 2. The entanglement entropy $S_{k}=S\left(\rho_{k}\right)=h\left(f_{k}\right)$ of a single particle mode $k$ with the rest of the system, for a level closest to the Fermi level $(k=\Omega / 2)$ (a) and most distant from the Fermi level $(k=1)(\mathrm{b})$, in the system of Fig. 1 Exact and BCS results are depicted.

Fermi-level (curve (a) in Fig. 2). Nonetheless, for sufficiently large $G$ all levels reached by the coupling become significantly affected.

In the strong pairing limit $G \gg \Omega \varepsilon, E(|\Psi\rangle)$ and all $h\left(f_{k}\right)$ saturate for $N=\Omega$, reaching their upper bounds: In this limit each term in the sum (22) will have the same weight, implying, for $N=\Omega$,

$$
\alpha_{\nu /(\Omega \varepsilon) \rightarrow \infty} \frac{1}{\sqrt{\left(\begin{array}{c}
\Omega \\
\Omega / 2
\end{array}\right)}} .
$$

Eq. (27) leads to $f_{k} \rightarrow 1 / 2$ and hence to $h\left(f_{k}\right) \rightarrow 1$ $\forall k$ (entailing maximum fluctuation $f_{k}\left(1-f_{k}\right) \rightarrow 1 / 4$ ), implying $E(|\Psi\rangle) \rightarrow 2 \Omega$.

The entanglement generated by the pairing correlations can also be seen at the bipartite level, by considering the bipartition of the full single particle space formed by the $\Omega$ single particle states $k$ and the $\Omega$ single particle states $\bar{k}\left(\mathcal{H}=\mathcal{H}_{\Omega} \oplus \mathcal{H}_{\bar{\Omega}}\right)$. For such partition the expression (22) is already the Schmidt decomposition of $|\Psi\rangle$, since each term in the sum involves orthogonal Slater Determinants at each side. The associated entanglement entropy is then

$$
E_{\Omega-\bar{\Omega}}(|\Psi\rangle)=-\sum_{\nu}\left|\alpha_{\nu}^{2}\right| \log _{2}\left|\alpha_{\nu}^{2}\right| .
$$

At the BCS level, this entropy is, remarkably, just half the one-body entropy (24) (see Eq. (430). In the exact result, this relation holds approximately for finite $\Omega$. Eq. (28) also increases with increasing $G / \varepsilon$ and saturates for $G /(\Omega \varepsilon) \rightarrow \infty$, where it reaches its upper bound compatible with a fixed $N: E_{\Omega-\bar{\Omega}}^{\max }=\log _{2}\left(\begin{array}{c}\Omega \\ \Omega / 2\end{array}\right)$ for $N=\Omega$. When scaled to its maximum value, $E_{\Omega-\bar{\Omega}} / E_{\Omega-\bar{\Omega}}^{\max }$ lies in fact very close to $E(|\Psi\rangle) /(2 \Omega)$, as seen in Fig. 3, Note also that for large $\Omega$, i.e. for a system with a large number $N=\Omega$ of particles, $\log _{2}\left(\begin{array}{c}\Omega \\ \Omega / 2\end{array}\right) \approx \Omega$ at leading order, which is half the saturation value of $E(|\Psi\rangle)$. This entails $E_{\Omega-\bar{\Omega}}=\frac{1}{2} E(|\Psi\rangle)$ in this limit, as in BCS.

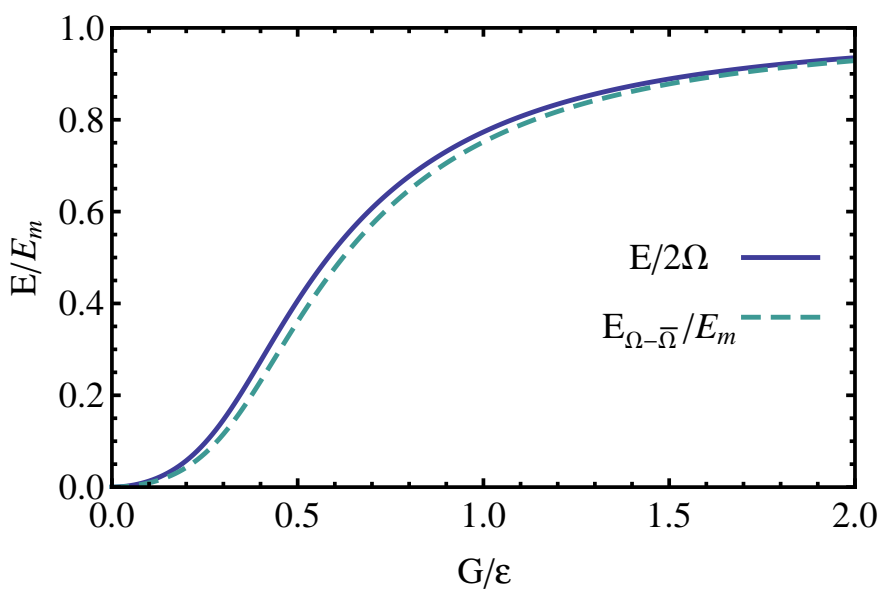

FIG. 3. The exact intensive one-body entanglement entropy (solid line) together with the bipartite $\Omega-\bar{\Omega}$ entanglement entropy (28) between all modes $k$ and their time-reversed partners $\bar{k}$ (dashed line), scaled to its maximum value, in the system of Fig. 1] In BCS these two quantities are identical.

\section{Entanglement of reduced states}

Regarding now the reduced state $\rho_{k \bar{k}}$ of a pair of modes $(k, \bar{k})$, just the outer $2 \times 2$ even parity block in Eq. (16), involving here the states $|0\rangle$ and $c_{k}^{\dagger} c_{\bar{k}}^{\dagger}|0\rangle$, will be nonzero, since the exact ground state contains no broken pairs and hence $\left\langle c_{k}^{\dagger} c_{k} c_{\bar{k}} c_{\bar{k}}^{\dagger}\right\rangle=0=\left\langle c_{\bar{k}}^{\dagger} c_{k}\right\rangle=\left\langle c_{k} c_{\bar{k}}\right\rangle$. Since $\left\langle c_{k}^{\dagger} c_{\bar{k}}^{\dagger} c_{\bar{k}} c_{k}\right\rangle=f_{k}$, this block will then be identical to (25), implying, $\forall k$,

$$
S\left(\rho_{k \bar{k}}\right)=S\left(\rho_{k}\right)=S\left(\rho_{\bar{k}}\right)=h\left(f_{k}\right) .
$$

Thus, there is a classical-like correlation between single particle modes $k$ and $\bar{k}$, captured by the mutual information

$$
I_{k \bar{k}}=S\left(\rho_{k}\right)+S\left(\rho_{\bar{k}}\right)-S\left(\rho_{k \bar{k}}\right)=h\left(f_{k}\right),
$$

which is then identical to the single mode entropy. Nonetheless, there is no entanglement between them since $\rho_{k \bar{k}}$ is diagonal in the basis $\left\{c_{k}^{\dagger} c_{\bar{k}}^{\dagger}|0\rangle,|0\rangle\right\}$ (this also shows that $E^{\mathrm{qsp}}\left(\rho_{k \bar{k}}\right)=E\left(\rho_{k \bar{k}}\right)=0$, as previously stated).

We can also omit states with broken pairs in the reduced density matrix of four single particle modes $\left(k \bar{k}, k^{\prime} \bar{k}^{\prime}\right), k \neq k^{\prime}$. The $(16 \times 16)$ matrix $\rho_{k \bar{k}} k^{\prime} \bar{k}^{\prime}$ then reduces to an effective $4 \times 4$ non-zero block $\rho_{k \bar{k}}^{r} k^{\prime} \bar{k}^{\prime}$, with support on the even number parity states $\left\{c_{k}^{\dagger} c_{\bar{k}}^{\dagger} c_{k^{\prime}}^{\dagger} c_{\bar{k}^{\prime}}^{\dagger}|0\rangle\right.$, 
$\left.c_{k}^{\dagger} c_{\bar{k}}^{\dagger}|0\rangle, c_{k^{\prime}}^{\dagger} c_{\bar{k}^{\prime}}^{\dagger}|0\rangle,|0\rangle\right\}$

$\rho_{k \bar{k} k^{\prime} \bar{k}^{\prime}}^{r}=\left(\begin{array}{cccc}\left\langle n_{k \bar{k}} n_{k^{\prime} \bar{k}^{\prime}}\right\rangle & 0 & 0 & 0 \\ 0 & \left\langle n_{k \bar{k}} \tilde{n}_{k^{\prime} \bar{k}^{\prime}}\right\rangle & \left\langle c_{k}^{\dagger} c_{\bar{k}}^{\dagger} c_{\bar{k}^{\prime}} c_{k^{\prime}}\right\rangle & 0 \\ 0 & \left\langle c_{k^{\prime}}^{\dagger} c_{\overline{k^{\prime}}}^{\dagger} c_{\bar{k}} c_{k}\right\rangle & \left\langle\tilde{n}_{k \bar{k}} n_{k^{\prime} \bar{k}^{\prime}}\right\rangle & 0 \\ 0 & 0 & 0 & \left\langle\tilde{n}_{k \bar{k}} \tilde{n}_{k^{\prime} \bar{k}^{\prime}}\right\rangle\end{array}\right)$

Here $n_{k \bar{k}}=c_{k}^{\dagger} c_{k} c_{\bar{k}}^{\dagger} c_{\bar{k}}, \tilde{n}_{k \bar{k}}=c_{k} c_{k}^{\dagger} c_{\bar{k}} c_{\bar{k}}^{\dagger}$ are non-zero iff the pair $(k, \bar{k})$ is fully occupied or empty respectively. In contrast with $\rho_{k \bar{k}}, \rho_{k \bar{k}} k^{\prime} \bar{k}^{\prime}$ will contain quantum correlations due to the nonzero off-diagonal element.

Its fermionic concurrence $C_{k k^{\prime}} \equiv C\left(\rho_{k \bar{k}} k^{\prime} \bar{k}^{\prime}\right)$, which determines the entanglement of formation $E\left(\rho_{k \bar{k}} k^{\prime} \bar{k}^{\prime}\right)=$ $E^{\mathrm{qsp}}\left(\rho_{k \bar{k} k^{\prime} \bar{k}^{\prime}}\right)$ through Eq. (19), becomes, using Eq. (20),

$$
C_{k k^{\prime}}=2 \operatorname{Max}\left[\left|\left\langle c_{k}^{\dagger} c_{\bar{k}}^{\dagger} c_{\bar{k}^{\prime}} c_{k^{\prime}}\right\rangle\right|-\sqrt{\left\langle n_{k \bar{k}} n_{k^{\prime} \bar{k}^{\prime}}\right\rangle\left\langle\tilde{n}_{k \bar{k}} \tilde{n}_{k^{\prime} \bar{k}^{\prime}}\right\rangle}, 0\right]
$$

which will be non-zero for $G>0$. Eq. (32) also represents here the bipartite concurrence [46] between modes $k \bar{k}$ and $k^{\prime} \bar{k}^{\prime}$, where each "side" is analogous to a qubit (it can be either empty or fully occupied in the ground state), forming together a two-qubit system (see Appendix C). The associated bipartite entanglement of formation is just $E_{k k^{\prime}}=E^{\mathrm{qsp}}\left(\rho_{k \bar{k}} k^{\prime} \bar{k}^{\prime}\right) / 4$. Thus, the fermionic entanglement of $\rho_{k \bar{k}} k^{\prime} \bar{k}^{\prime}$ can be directly identified here with a bipartite entanglement. It is also verified, by simple use of Wick's theorem, that $C_{k k^{\prime}}$ vanishes identically in the BCS approximation (see next section), so that this entanglement lies strictly beyond the standard BCS description, in contrast with previous quantities. As stated before, $C_{k k^{\prime}}$ vanishes for all fermionic gaussian states, which include in particular the BCS ground state. A finite concurrence requires sufficiently strong two-body correlations.

As seen in Fig. 4, $E_{k k^{\prime}}$ exhibits, remarkably, a prominent peak in the vicinity of the BCS superconducting transition region $G \approx G_{c}$ for the pair of levels closest to the Fermi level, becoming then small for $G \gg G_{c}$. This peak is obviously also present in the concurrence $C_{k k^{\prime}}\left(E_{k k^{\prime}}\right.$ is just an increasing function of $\left.C_{k k^{\prime}}\right)$, and its height rapidly decays for levels $k, k^{\prime}$ not too close to the Fermi surface, disappearing for distant levels.

The previous behavior can be understood by analyzing first the strong superconducting limit $G /(\Omega \varepsilon) \rightarrow \infty$, where $\rho_{k \bar{k}}^{r} k^{\prime} \bar{k}^{\prime}$ will be independent of $k, k^{\prime}$, according to Eq. (27): The diagonal terms $\left\langle n_{k \bar{k}} n_{k^{\prime} \bar{k}^{\prime}}\right\rangle$ and $\left\langle\tilde{n}_{k \bar{k}} \tilde{n}_{k^{\prime} \bar{k}^{\prime}}\right\rangle$ in (31), which are the probabilities of finding both pairs fully occupied or empty become, for $N=\Omega / 2$,

$$
\left\langle n_{k \bar{k}} n_{k^{\prime} \bar{k}^{\prime}}\right\rangle=\left\langle\tilde{n}_{k \bar{k}} \tilde{n}_{k^{\prime} \bar{k}^{\prime}}\right\rangle=\frac{\left(\begin{array}{c}
\Omega-2 \\
\Omega / 2
\end{array}\right)}{\left(\begin{array}{l}
\Omega \\
\Omega / 2
\end{array}\right)}=\frac{\Omega-2}{4(\Omega-1)},
$$

while all elements of the inner block become equal,

$$
\left\langle n_{k \bar{k}} \tilde{n}_{k^{\prime} \bar{k}^{\prime}}\right\rangle=\left\langle\tilde{n}_{k \bar{k}} n_{k^{\prime} \bar{k}^{\prime}}\right\rangle=\left\langle c_{k}^{\dagger} c_{\bar{k}}^{\dagger} c_{\bar{k}^{\prime}} c_{k^{\prime}}\right\rangle=\frac{\left(\begin{array}{c}
\Omega-2 \\
\Omega / 2-1
\end{array}\right)}{\left(\begin{array}{c}
\Omega \\
\Omega / 2
\end{array}\right)}=\frac{\Omega}{4(\Omega-1)},
$$

implying it will have a single non-zero eigenvalue $\frac{\Omega}{2(\Omega-1)}$.

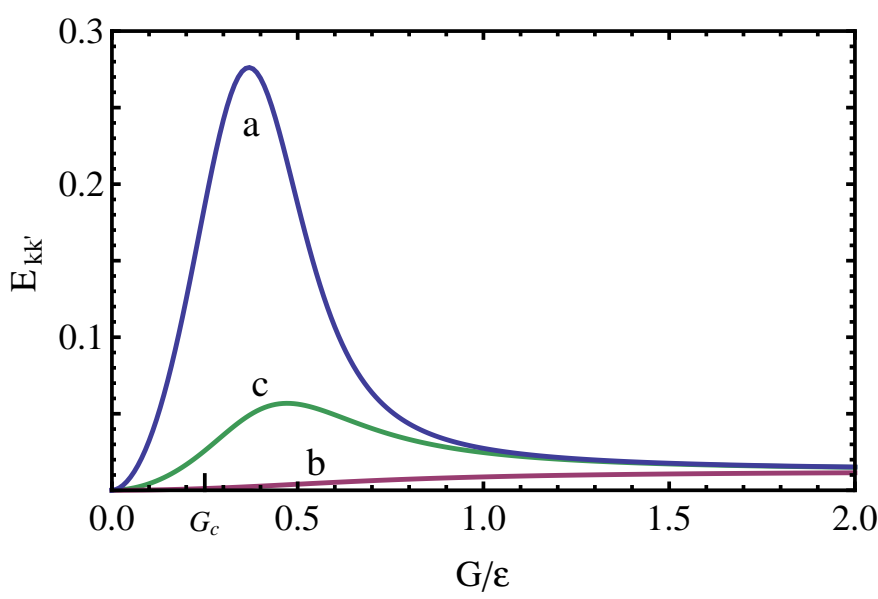

FIG. 4. Entanglement of formation $E_{k k^{\prime}}$ determined by the concurrence (32) between the modes $k \bar{k}$ and $k^{\prime} \bar{k}^{\prime}$, for pairs closest $\left(k=\Omega / 2, k^{\prime}=k+1\right)$ (a) and most distant $\left(k=1, k^{\prime}=\right.$ $\Omega$ ) (b) to the Fermi level, and also for pairs next to closest $\left(k=\Omega / 2-1, k^{\prime}=k+3\right)$ (c), in the system of Fig. 1. The BCS result for this quantity vanishes identically. The peak in (a) occurs close to the BCS superconducting transition.

With Eqs. (33)-(34), Eq. (32) leads in this limit to

$$
C_{k k^{\prime}}=\frac{1}{\Omega-1}
$$

$\forall k \neq k^{\prime}$, decreasing as $\Omega^{-1}$ for large $\Omega$ (i.e, for a large number $N=\Omega$ of fermions) and implying $E_{k k^{\prime}} \approx$ $\frac{1}{2} \Omega^{-2} \log _{2}(2 \Omega \sqrt{e})$. Eq. (35) is in agreement with the result for fully connected systems [48] and the monogamy property of the concurrence [49, 50] $\left(\sum_{k^{\prime} \neq k} C_{k k^{\prime}}^{2} \leq\right.$ $\left.C_{k,\left\{k^{\prime} \neq k\right\}}^{2}\right)$. All $\rho_{k \bar{k} k^{\prime} \bar{k}^{\prime}}^{r}$ become equally entangled in this limit for $k \neq k^{\prime}$, implying that $C_{k k^{\prime}}$ should scale with $\Omega^{-1}$ for large $\Omega$.

Thus, for not too small $\Omega$, monogamy prevents a significant entanglement between pairs $k \bar{k}$ and $k^{\prime} \bar{k}^{\prime}$ in the strong superconducting regime. In contrast, at the onset of superconductivity $\left(G \approx G_{c}\right.$ ) just the levels $k, k^{\prime}$ closest to the Fermi level are affected by the pairing correlations, originating the initial increase and ensuing peak in the corresponding $C_{k k^{\prime}}$ and $E_{k k^{\prime}}$. As $G$ increases further, $C_{k k^{\prime}}$ becomes appreciable for an increasing number of pairs $k \neq k^{\prime}$ around the Fermi level and the highest $C_{k k^{\prime}}$ must then decrease to comply with monogamy requirements. For large $\Omega, C_{k k^{\prime}}$ is then significant only for $k, k^{\prime}$ close to the Fermi level and just at the transition region, rather than at the strong superfluid regime.

In the latter, correlations between modes $k \bar{k}$ and $k^{\prime} \bar{k}^{\prime}$ in the reduced state $\rho_{k \bar{k}} k^{\prime} \bar{k}^{\prime}$ do exist, but lead just to a finite mutual information $I_{k k^{\prime}}$ and finite quantum discord $D_{k k^{\prime}}$ (see appendix C), as shown in Fig. [5] In the limit $G /(\Omega \varepsilon) \rightarrow \infty$, Eqs. (33)- (34) lead to $S\left(\rho_{k \bar{k} k^{\prime} \bar{k}^{\prime}}^{r}\right) \approx \frac{1}{2}(3-$ 

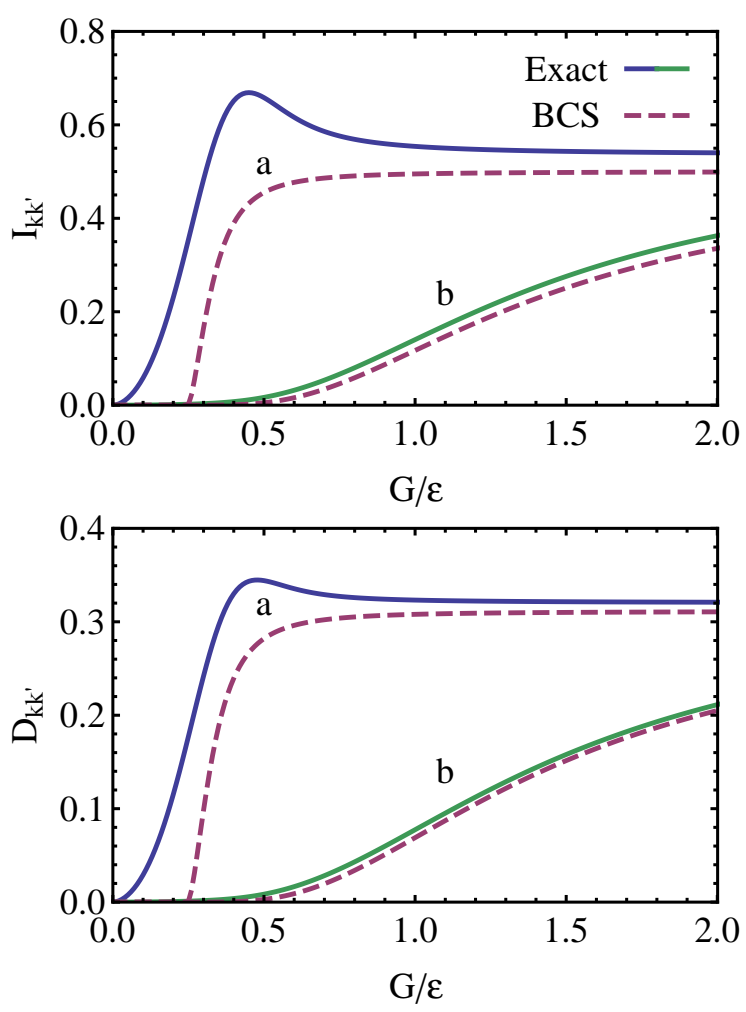

FIG. 5. The mutual information (36) (top) and the quantum discord (bottom) between modes $k \bar{k}$ and $k^{\prime} \bar{k}^{\prime}$, for pairs closest (a) and most distant (b) to the Fermi level, in the system of Fig. 1. Both quantities are significant in the superconducting phase. The BCS estimate is now non-vanishing for $G>G_{c}$.

$\Omega^{-1}$ ) for large $\Omega$, implying that the mutual information

$$
\begin{aligned}
I_{k k^{\prime}} & \equiv S\left(\rho_{k \bar{k}}\right)+S\left(\rho_{k^{\prime} \bar{k}^{\prime}}\right)-S\left(\rho_{k \bar{k} k^{\prime} \bar{k}^{\prime}}\right) \\
& =h\left(f_{k}\right)+h\left(f_{k^{\prime}}\right)-S\left(\rho_{k \bar{k} k^{\prime} \bar{k}^{\prime}}^{r}\right),
\end{aligned}
$$

approaches in this limit a finite common value $\approx \frac{1}{2}(1+$ $\Omega^{-1}$ ) for all $k \neq k^{\prime}$. Both $I_{k k^{\prime}}$ and $D_{k k^{\prime}}$ are initially obviously larger for pairs $k, k^{\prime}$ close to the fermi level, in which case they develop a moderate peak at the transition region, but remain significant for $G \gg G_{c}$ since they are not restricted by a monogamy property [42]. And in contrast with $E_{k k^{\prime}}$, they can be correctly estimated through BCS. The finite value of the discord (whose calculation details and asymptotic expression are discussed in Appendix $[$ indicates that in the strong pairing regime, the correlations between pairs $k \bar{k}$ and $k^{\prime} \bar{k}^{\prime}$, while not leading to a significant entanglement of the reduced state, are not fully classical either. As seen from Eqs. (31)(34), $\rho_{k \bar{k}, k^{\prime} \bar{k}^{\prime}}^{r}$ does not become diagonal in a product basis, having instead a maximally entangled non-degenerate eigenstate in the inner block, which leads to the previous non-classical effect (finite discord).

In the smallest non-trivial case $\Omega=2, \rho_{k \bar{k} k^{\prime} \bar{k}^{\prime}}=|\Psi\rangle\langle\Psi|$ becomes pure $\left(k=1, k^{\prime}=2\right)$. Hence, the discord coincides with $E_{k k^{\prime}}$, which in turn becomes proportional the one-body entanglement entropy and also the entropy (28): $D_{k k^{\prime}}=E_{k k^{\prime}}=E_{\Omega-\bar{\Omega}}=E(|\Psi\rangle) / 4=h\left(f_{k}\right)=$ $h\left(f_{k}^{\prime}\right)$, where $f_{k}$ and $f_{k^{\prime}}=1-f_{k}$ are the (two-fold degenerate) eigenvalues of $\rho^{\text {sp }}$ (see end of Appendix C). All previous measures then collapse to a single value.

\section{B. The BCS description}

\section{Standard treatment}

As is well known, the BCS approximation to the ground state of Hamiltonian (21) relies on a state of the form [45, 51]

$$
|\mathrm{BCS}\rangle=\left[\prod_{k}\left(u_{k}+v_{k} c_{k}^{\dagger} c_{\bar{k}}^{\dagger}\right)\right]|0\rangle,
$$

for even $N$, where $\left|u_{k}^{2}\right|+\left|v_{k}^{2}\right|=1$. Such state is the vacuum of quasiparticle fermion operators

$$
a_{k}=u_{k} c_{k}-v_{k} c_{\bar{k}}^{\dagger}, \quad a_{\bar{k}}=v_{k} c_{k}^{\dagger}+u_{k} c_{\bar{k}},
$$

satisfying $a_{k}|\mathrm{BCS}\rangle=a_{\bar{k}}|\mathrm{BCS}\rangle=0$ together with fermion anticommutation relations. The coefficients minimizing $\langle H\rangle=\langle\mathrm{BCS}|H| \mathrm{BCS}\rangle$ under a fixed $\langle N\rangle=2 \sum_{k}\left|v_{k}^{2}\right|$ constraint can be chosen real nonnegative, and are given by ${ }_{v_{k}}^{u_{k}}=\sqrt{\frac{\lambda_{k} \pm \tilde{\varepsilon}_{k}}{2 \lambda_{k}}}$, where $\lambda_{k}=\sqrt{\tilde{\varepsilon}_{k}^{2}+\Delta^{2}}$ are the quasiparticle energies, $\tilde{\varepsilon}_{k}=\varepsilon_{k}-\mu$, with $\mu$ the chemical potential and $\Delta=G \sum_{k}\left\langle c_{\bar{k}} c_{k}\right\rangle$ is the BCS gap (we have dismissed minor effects in $\tilde{\varepsilon}_{k}$ stemming from self-energy terms). For $\langle N\rangle=\Omega$ and a uniformly spaced spectrum, $\mu=\frac{1}{\Omega} \sum_{k} \varepsilon_{k}$.

As $\left\langle c_{\bar{k}} c_{k}\right\rangle=u_{k} v_{k}=\Delta /\left(2 \lambda_{k}\right), \Delta$ is determined by the gap equation

$$
\Delta=G \Delta \sum_{k} \frac{1}{2 \lambda_{k}} .
$$

The superconducting phase corresponds to the nontrivial solution $\Delta>0$, which arises for $G>G_{c}$ with

$$
G_{c}=\frac{2}{\sum_{k} \frac{1}{\left|\tilde{\varepsilon}_{k}\right|}} \approx \frac{\varepsilon}{\ln (\Omega / 2)+\gamma},
$$

where the last expression holds for large $\Omega$ in the equally spaced case $\left(\gamma=-\frac{\Gamma^{\prime}[1 / 2]}{\Gamma[1 / 2]} \approx 1.96\right)$. For $G<G_{c}, \Delta=0$.

While $E^{\text {qsp }}(|\mathrm{BCS}\rangle)=0 \forall G$, as $|\mathrm{BCS}\rangle$ is a quasiparticle vacuum, the one-body entanglement entropy

$$
E(|\mathrm{BCS}\rangle)=\operatorname{tr}\left[h\left(\rho_{\mathrm{BCS}}^{\mathrm{sp}}\right)\right]=2 \sum_{k} h\left(f_{k}\right),
$$

is finite for $G>G_{c}$ and provides an excellent approximation to the exact $E(|\Psi\rangle)=E^{\mathrm{qsp}}(|\Psi\rangle)$ in the superconducting phase, as seen in Fig. 1. Here $\rho_{\mathrm{BCS}}^{\mathrm{sp}}=$ $\left\langle\mathrm{BCS}\left|\mathbb{1}-\boldsymbol{c c}^{\dagger}\right| \mathrm{BCS}\right\rangle$ is the BCS single particle density matrix, which is diagonal in the unperturbed single particle basis, with eigenvalues $f_{k}=\left\langle c_{k}^{\dagger} c_{k}\right\rangle=\left\langle c_{\bar{k}}^{\dagger} c_{\bar{k}}\right\rangle$ given by

$$
f_{k}=\left|v_{k}^{2}\right|=\frac{1}{2}\left(1-\frac{\tilde{\varepsilon}_{k}}{\lambda_{k}}\right) .
$$


For $G<G_{c}, v_{k}^{2}=1$ (0) for levels below (above) the Fermi level and (41) vanishes, whereas for $G>G_{c}, v_{k}^{2} \in$ $(0,1)$, smoothing the Fermi surface and leading to a finite value of (41). This indicates the departure of (37) from a standard Slater determinant. For $G /(\Omega \varepsilon) \rightarrow \infty$ and $N=$ $\Omega$, Eq. (39) leads to $\Delta \approx G \Omega / 2$ and $v_{k}^{2} \approx \frac{1}{2}\left(1-\tilde{\varepsilon}_{k} / \Delta\right)$, implying $E(|\mathrm{BCS}\rangle) \approx 2 \Omega\left[1-\frac{\sum_{k} \tilde{\varepsilon}_{k}^{2}}{2 \Omega \Delta^{2} \ln 2}\right]$, which saturates in the limit.

Eq. (42) also provides a good approximation to the single mode entropies $S\left(\rho_{k}\right)=h\left(f_{k}\right)$, as seen in Fig. 2 As stated before, each term $h\left(f_{k}\right)$ is an increasing function of the occupation number fluctuation $f_{k}\left(1-f_{k}\right)$, which in BCS becomes $u_{k}^{2} v_{k}^{2}$. And the quadratic onebody entanglement entropy $S_{2}\left(\rho^{\mathrm{sp}}\right)=4 \operatorname{tr}\left[\rho^{\mathrm{sp}}\left(\mathbb{1}-\rho^{\mathrm{sp}}\right)\right]=$ $8 \sum_{k} u_{k}^{2} v_{k}^{2}$ is in BCS just twice the total number fluctuation $\left\langle N^{2}\right\rangle-\langle N\rangle^{2}$ 45]. Of course, this relation is not valid in the exact ground state, for which the number fluctuation is strictly zero.

The BCS state (37) does not have a fixed fermion number but has a definite (positive) number parity. It is in fact of the same form (22) with $\alpha_{\nu}=\prod_{k} v_{k}^{n_{k}^{\nu}} u_{k}^{1-n_{k}^{\nu}}$ and $n_{k}^{\nu}=0,1$ independent variables, such that $\nu=1, \ldots, 2^{\Omega}$. Thus, the entanglement entropy (28) between all modes $k$ and all modes $\bar{k}$ reduces to the entropy of a product of $\Omega$ independent density operators with eigenvalues $\left|v_{k}^{2}\right|=f_{k}$ and $\left|u_{k}^{2}\right|=1-f_{k}$. Hence,

$$
E_{\Omega-\bar{\Omega}}(|\mathrm{BCS}\rangle)=\sum_{k} h\left(f_{k}\right)=\frac{1}{2} E(|\mathrm{BCS}\rangle) .
$$

A similar relation holds approximately in the exact result (Fig. 3). An entropy similar to (43) was defined in [20] for the BCS state and analyzed in the continuous limit.

Considering now the reduced state of levels $(k \bar{k})$, BCS leads (using Wick's theorem [45]) to $\left\langle c_{k}^{\dagger} c_{k} c_{\bar{k}} c_{\bar{k}}^{\dagger}\right\rangle=$ $\left\langle c_{k}^{\dagger} c_{k}\right\rangle\left\langle c_{\bar{k}} c_{\bar{k}}^{\dagger}\right\rangle-\left\langle c_{k}^{\dagger} c_{\bar{k}}^{\dagger}\right\rangle\left\langle c_{\bar{k}} c_{k}\right\rangle=v_{k}^{2} u_{k}^{2}-\left(u_{k} v_{k}\right)^{2}=0$ and $\left\langle c_{k}^{\dagger} c_{\bar{k}}^{\dagger} c_{\bar{k}} c_{k}\right\rangle=v_{k}^{4}+\left(u_{k} v_{k}\right)^{2}=f_{k}$, as in the exact case. Hence, Eqs. (29)-(30) remain valid in BCS with $f_{k}$ given by (42).

Differences arise, however, in the four level density matrix (31), since Wick's theorem implies that all quantities will be a function of the $f_{k}$. For $k \neq k^{\prime}$ we have $\left\langle n_{k \bar{k}} n_{k^{\prime} \bar{k}^{\prime}}\right\rangle=\left\langle n_{k \bar{k}}\right\rangle\left\langle n_{k^{\prime} \bar{k}^{\prime}}\right\rangle,\left\langle n_{k \bar{k}} \tilde{n}_{k^{\prime} \bar{k}^{\prime}}\right\rangle=\left\langle n_{k \bar{k}}\right\rangle\left\langle\tilde{n}_{k^{\prime} \bar{k}^{\prime}}\right\rangle$ and $\left\langle\tilde{n}_{k \bar{k}} \tilde{n}_{k^{\prime} \bar{k}^{\prime}}\right\rangle=\left\langle\tilde{n}_{k \bar{k}}\right\rangle\left\langle\tilde{n}_{k^{\prime} \bar{k}^{\prime}}\right\rangle$, with $\left\langle n_{k \bar{k}}\right\rangle=v_{k}^{2}=f_{k}$, $\left\langle\tilde{n}_{k}\right\rangle=u_{k}^{2}=1-f_{k} \equiv \tilde{f}_{k}$ and $\left\langle c_{k}^{\dagger} c_{\bar{k}}^{\dagger} c_{\bar{k}^{\prime}} c_{k^{\prime}}\right\rangle=u_{k} v_{k} u_{k^{\prime}} v_{k^{\prime}}$. Hence, in BCS Eq. (31) becomes

$$
\rho_{k \bar{k} k^{\prime} \bar{k}^{\prime}}^{r \mathrm{BCS}}=\left(\begin{array}{cccc}
f_{k} f_{k^{\prime}} & 0 & 0 & 0 \\
0 & f_{k} \tilde{f}_{k^{\prime}} & \sqrt{f_{k} \tilde{f}_{k} f_{k^{\prime}} \tilde{f}_{k^{\prime}}} & 0 \\
0 & \sqrt{f_{k} \tilde{f}_{k} f_{k^{\prime}} \tilde{f}_{k^{\prime}}} & \tilde{f}_{k} f_{k^{\prime}} & 0 \\
0 & 0 & 0 & \tilde{f}_{k} \tilde{f}_{k^{\prime}}
\end{array}\right) .
$$

It then has always just three non-zero eigenvalues $\left(f_{k} f_{k^{\prime}}\right.$, $\tilde{f}_{k} \tilde{f}_{k^{\prime}}$ and $\left.f_{k} \tilde{f}_{k^{\prime}}+\tilde{f}_{k} f_{k^{\prime}}\right)$, which in the exact state occurs exactly only for $G /(\Omega \varepsilon) \rightarrow \infty$. These expressions lead to $\left|\left\langle c_{k}^{\dagger} c_{\bar{k}}^{\dagger} c_{\bar{k}^{\prime}} c_{k^{\prime}}\right\rangle\right|=\sqrt{\left\langle n_{k \bar{k}} n_{k^{\prime} \bar{k}^{\prime}}\right\rangle\left\langle\tilde{n}_{k \bar{k}} \tilde{n}_{k^{\prime} \bar{k}^{\prime}}\right\rangle}$ and hence to
$C_{k k^{\prime}}=0 \forall \Delta$ and $k \neq k^{\prime}$, as previously stated. BCS cannot reproduce the concurrence (32) since the latter vanishes in any gaussian state, and hence for any $|\Psi\rangle$ which is a Slater determinant or quasiparticle vacuum, like the BCS state (37). A finite concurrence requires sufficiently strong two-body correlations, in order to have a positive difference in (32).

Nonetheless, BCS still leads to a good estimate of $S\left(\rho_{k \bar{k} k^{\prime} \bar{k}^{\prime}}\right)$ and of both the mutual information $I_{k k^{\prime}}$ and quantum discord $D_{k k^{\prime}}$ in the superconducting phase $G>$ $G_{c}$, as seen in Fig. 5. Moreover, for $G /(\Omega \varepsilon) \rightarrow \infty, f_{k} \rightarrow$ $1 / 2 \forall k$, and the exact limits $I_{k k^{\prime}}=1 / 2, S\left(\rho_{k k^{\prime}}\right)=3 / 2$ and $D_{k k^{\prime}}=\frac{3}{2}-3 \frac{\log _{2} 3}{4}$ (see Appendix C) are obtained for large $\Omega$.

\section{Number projected treatment}

One could now ask how the finite value of the concurrence $C_{k k^{\prime}}$ can be predicted in a BCS-based scheme. The answer lies, of course, in the number projected BCS approximation [45], based on the state

$$
P_{N}|\mathrm{BCS}\rangle \propto \sum_{\nu}\left[\prod_{k} v_{k}^{n_{k}^{\nu}} u_{k}^{1-n_{k}^{\nu}}\left(c_{k}^{\dagger} c_{\bar{k}}^{\dagger}\right)^{n_{k}^{\nu}}\right]|0\rangle,
$$

where $P_{N}=\frac{1}{2 \pi} \int_{0}^{2 \pi} e^{-i \phi(\hat{N}-N)} d \phi$ is the projector onto fixed (even) fermion number $N, n_{k}^{\nu}=0,1$ and now $\sum_{k} n_{k}^{\nu}=N / 2$, with $\nu=1, \ldots,\left(\begin{array}{c}\Omega \\ N / 2\end{array}\right)$ (the $n_{k}^{\nu}$ are no longer independent variables). The state (45) has the same form as the exact state (22), but with specified coefficients $\alpha_{\nu}=\prod_{k} v_{k}^{n_{k}^{\nu}} u_{k}^{1-n_{k}^{\nu}}$.

While projection after variation already improves BCS in the superconducting phase, projection before variation can properly describe also the normal sector $G<G_{c}$, where standard BCS estimates vanish for all correlation measures. We consider here a simple approach where the form of the coefficients $u_{k}$ and $v_{k}$ in (45) is the same as in standard BCS, but $\Delta$ is left as a variational parameter to be determined from the minimization of the projected average energy $\langle H\rangle_{N}=\frac{\left\langle\mathrm{BCS}\left|P_{N} H\right| \mathrm{BCS}\right\rangle}{\left\langle\mathrm{BCS}\left|P_{N}\right| \mathrm{BCS}\right\rangle}$. Full self-consistent methods can also be employed [52].

As seen in Fig. 6, such an approach is sufficient to predict a finite concurrence $C_{k k^{\prime}}$, which fairly reproduces the exact result, including the peak for pairs $k \bar{k}, k^{\prime} \bar{k}^{\prime}$ close to the fermi level. The essential reason is that for $\Delta>0$, the projected state (45) is no longer gaussian, i.e. it is not a quasiparticle vacuum nor a Slater determinant, and Wick's theorem no longer holds. It contains two-body correlations and has in fact a very high overlap with the exact ground state (22).

The effective $\Delta$ obtained with projection before variation is positive for all $G>0$ and exhibits a smooth increase with increasing $G$, so that (45) will also lead to quite accurate estimates of all other quantum correlation measures shown in Figs. 10.3 and 5 including the interval $0<G \leq G_{c}$, as seen in the bottom panel of 

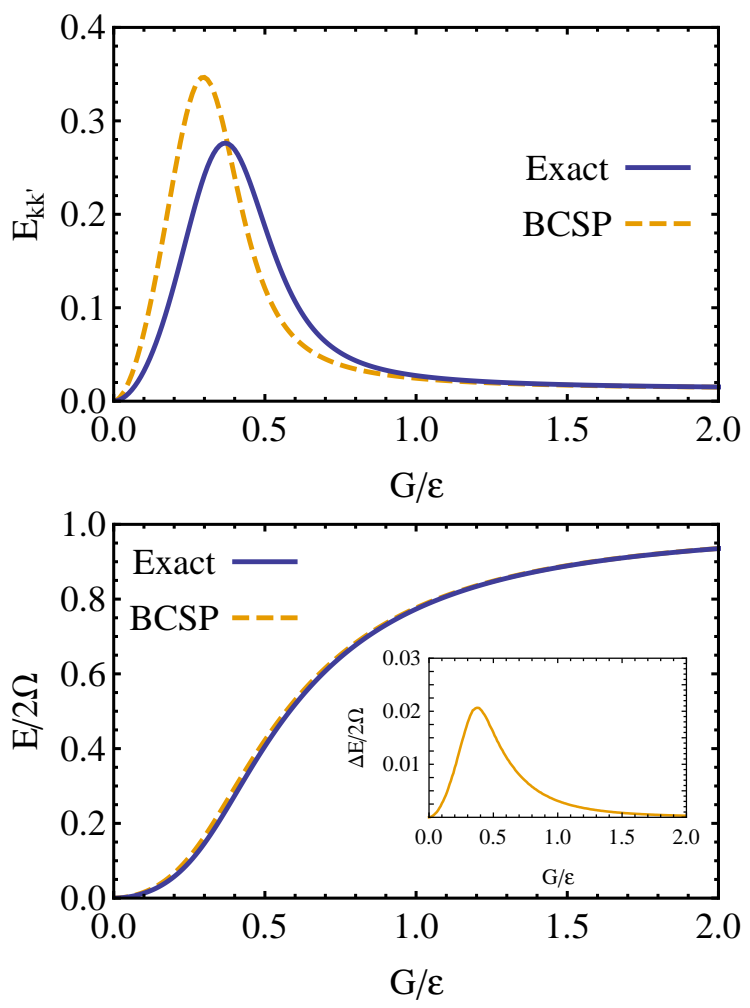

FIG. 6. Top: Entanglement of formation $E_{k k^{\prime}}$ between modes $k \bar{k}$ and $k^{\prime} \bar{k}^{\prime}$ for pairs closest to the Fermi level according to exact and projected BCS results, in the system of Fig. 1. Bottom: The corresponding one body entanglement entropy. The projected BCS result is accurate for all values of $G$, although the difference $\frac{\Delta E}{2 \Omega}=\frac{\left(E_{\mathrm{BCSP}}-E_{\text {Exact }}\right)}{2 \Omega}$, depicted in the inset, is also maximum at the transition region.

Fig. 6 for the one-body entanglement entropy. It is also noticed that the transition region $G \approx G_{c}$, where $C_{k k^{\prime}}$ exhibits its peak, is precisely that where the discrepancy between the exact and the projected BCS predictions is most significant, as seen in the inset.

\section{CONCLUSIONS}

We have analyzed in detail the behavior of general fermionic entanglement measures in the exact ground state of a finite superconducting system. The onebody entanglement entropy, which represents the minimum distance (as measured by the relative entropy) to a fermionic gaussian state, is seen to be here a close indicator of pairing correlations, saturating in the strong coupling limit and behaving like a scaled BCS gap. It is practically proportional to the bipartite entanglement entropy between all single particle modes $k$ and their time reversed partners $\bar{k}$, being exactly proportional at the BCS level. BCS provides in fact a good estimation of these entropies in the whole superconducting phase.

In contrast, the entanglement of a subset of fermionic modes, determined in general by a mixed reduced state with no fixed fermion number, can exhibit a quite different behavior. The first non-trivial case was shown to be that of four single particle modes $k, \bar{k}, k^{\prime}, \bar{k}^{\prime}$, whose entanglement of formation can be evaluated through the fermionic concurrence and can be interpreted as a bipartite mode entanglement. This entanglement vanishes identically in BCS as well as in any fermionic gaussian state. In the exact ground state it shows instead a peak in the vicinity of the superconducting transition region for single particle states $k, k^{\prime}$ close to the Fermi level, which are those most affected by the coupling at the onset of the transition, possessing then a larger occupation number fluctuation in this region. The concurrence becomes then small in the strong coupling regime for not too small $\Omega$ due to monogamy restrictions. Hence, it is here an indicator of the transition, reflecting the increased complexity of the exact ground state in this region. It requires at least a number projected BCS treatment for its approximate description. We have also shown that while not significantly entangled, these four modes do remain correlated in the strong coupling regime, exhibiting there a finite mutual information and quantum discord, due the non-zero off diagonal terms in the density matrix, and showing for this reason a less noticeable peak at the transition region. The present results provide then new insights into the relation between fermionic entanglement and superconducting correlations.

\section{ACKNOWLEDGMENTS}

We acknowledge support from $\mathrm{CIC}(\mathrm{RR})$ and CONICET (MDT, NG), of Argentina, and CONICET Grant PIP 112201501-00732. Discussions with Prof. J.M. Matera are also acknowledged.

\section{Appendix A: Minimum relative entropy}

Given two density operators $\rho, \rho^{\prime}$ for a given system, the relative entropy (86) can be written as [43, 44]

$$
S\left(\rho \| \rho^{\prime}\right)=-\operatorname{Tr}\left[\rho \log _{2} \rho^{\prime}\right]-S(\rho),
$$

where $S(\rho)=-\operatorname{Tr}\left[\rho \log _{2} \rho\right]$ is the von Neumann entropy. It satisfies $S\left(\rho \| \rho^{\prime}\right) \geq 0$, with $S\left(\rho \| \rho^{\prime}\right)=0$ iff $\rho=\rho^{\prime}$ [43]. Let us now consider a $\rho^{\prime}$ of the form

$$
\rho^{\prime}=Z^{-1} \exp \left[-\sum_{\nu=1}^{m} \lambda_{\nu} O_{\nu}\right]
$$

where $Z=\operatorname{Tr} \exp \left[-\sum_{\nu} \lambda_{\nu} O_{\nu}\right]$ and $\left\{O_{\nu}, \nu=1, \ldots, m\right\}$ is an arbitrary set of $m$ linearly independent operators (hermitian or comprising both $O_{\nu}$ and $O_{\nu}^{\dagger}$ ). This form of $\rho^{\prime}$ is that which maximizes the entropy $S\left(\rho^{\prime}\right)$ subject to the constraint of fixed expectation values $\left\langle O_{\nu}\right\rangle, \nu=$ $1, \ldots, m$. It is easy to show that for fixed $\rho$,

$$
\operatorname{Min}_{\left\{\lambda_{\nu}\right\}} S\left(\rho \| \rho^{\prime}\right)=S\left(\rho^{\prime}\right)-S(\rho),
$$


with the minimum reached for those $\lambda_{\nu}$ satisfying

$$
\operatorname{Tr}\left[\rho^{\prime} O_{\nu}\right]=\operatorname{Tr}\left[\rho O_{\nu}\right], \quad \nu=1, \ldots, m,
$$

i.e., for that $\rho^{\prime}$ which reproduces the expectation values determined by $\rho$ of all operators $O_{\nu}$ of the chosen set. Proof: Setting $\left\langle O_{\nu}\right\rangle_{\rho} \equiv \operatorname{Tr}\left[\rho O_{\nu}\right]$, we obtain, from Eqs. (A1)- (A2),

$$
S\left(\rho \| \rho^{\prime}\right)=\frac{1}{\ln 2}\left(\sum_{\nu=1}^{k} \lambda_{\nu}\left\langle O_{\nu}\right\rangle_{\rho}+\ln Z\right)-S(\rho) .
$$

As $\frac{\partial \ln Z}{\partial \lambda_{\nu}}=-\left\langle O_{\nu}\right\rangle_{\rho^{\prime}}$, equations $\frac{\partial}{\partial \lambda_{\nu}} S\left(\rho \| \rho^{\prime}\right)=0$ lead to

$$
\left\langle O_{\nu}\right\rangle_{\rho^{\prime}}=\left\langle O_{\nu}\right\rangle_{\rho}, \quad \nu=1, \ldots, m
$$

in which case Eq. (A5) reduces to Eq. (A3).

It then follows that $S\left(\rho^{\prime}\right) \geq S(\rho)$, with $S\left(\rho^{\prime}\right)=S(\rho)$ iff $\rho^{\prime}=\rho$. The minimum relative entropy is then a measure of the information contained in $\rho$ that cannot be contained in any operator of the form A2 . If $\rho$ is pure and the operators $O_{\nu}$ comprise the full set of one-body operators $c_{i}^{\dagger} c_{j}$, (A3) leads to Eq. (10) provided traces are taken in the grand canonical ensemble. Similarly, if the $O_{\nu}$ also include the operators $c_{i} c_{j}$ and $c_{i}^{\dagger} c_{j}^{\dagger}$, (A33) leads to Eq. (13) (again in the full grand canonical ensemble).

\section{Appendix B: Fermionic concurrence of four single particle states}

Labeling the four single particle states $i, j, k, l$ as $1,2,3,4$, and setting $|\overline{0}\rangle \equiv c_{1}^{\dagger} c_{2}^{\dagger} c_{3}^{\dagger} c_{4}^{\dagger}|0\rangle$, with $|0\rangle$ the fermionic vacuum, the operator $T$ in $R\left(\rho_{i j k l}\right)$ in Eq. (20) is represented, in the basis $\left\{|0\rangle, c_{1}^{\dagger} c_{2}^{\dagger}|0\rangle, c_{1}^{\dagger} c_{3}^{\dagger}|0\rangle, c_{1}^{\dagger} c_{4}^{\dagger}|0\rangle,-|\overline{0}\rangle, c_{2} c_{1}|\overline{0}\rangle, c_{3} c_{1}|\overline{0}\rangle, c_{4} c_{1}|\overline{0}\rangle\right\}$, by the matrix [27]

$$
T=\left(\begin{array}{cc}
0 & I_{4} \\
I_{4} & 0
\end{array}\right)
$$

The same matrix holds for an odd parity state $\rho_{i j k l}$ in the basis $\left\{c_{1}^{\dagger}|0\rangle, c_{2}^{\dagger}|0\rangle, c_{3}^{\dagger}|0\rangle, c_{4}^{\dagger}|0\rangle, c_{1}|\overline{0}\rangle, c_{2}|\overline{0}\rangle, c_{3}|\overline{0}\rangle, c_{4}|\overline{0}\rangle\right\}$.

\section{Appendix C: Four modes reduced states as two-qubit states and quantum discord}

The ground state in (22) is a superposition of states where pairs of modes $k \bar{k}$ are either fully occupied or empty. Following ref. [31], we could think of such pairs as even-parity qubits and use this representation to see the reduced state (31) of the four modes $k \bar{k}, k^{\prime} \bar{k}^{\prime}$, as a mixed two-qubit state. From lemma 1 of [31] it then follows that the fermionic concurrence (32) is the Wootters concurrence [46] of these two qubits.
Furthermore, fermion operators analogous to the Pauli matrices can be introduced for these qubits, so that any local operation can be described in terms of them:

$$
\begin{aligned}
& \tilde{\sigma}_{k}^{x}=c_{k}^{\dagger} c_{\bar{k}}^{\dagger}+c_{\bar{k}} c_{k} \\
& \tilde{\sigma}_{k}^{y}=-i\left(c_{k}^{\dagger} c_{\bar{k}}^{\dagger}-c_{\bar{k}} c_{k}\right) \\
& \tilde{\sigma}_{k}^{z}=c_{k}^{\dagger} c_{k}+c_{\bar{k}}^{\dagger} c_{\bar{k}}-1 .
\end{aligned}
$$

It is verified that these operators satisfy $\left[\tilde{\sigma}_{k}^{\mu}, \tilde{\sigma}_{k^{\prime}}^{\nu}\right]=$ $2 i \delta_{k k^{\prime}} \epsilon_{\mu \nu \gamma} \tilde{\sigma}_{k}^{\gamma}$ and $\left(\tilde{\sigma}_{k}^{\mu}\right)^{2}|\psi\rangle_{k}=|\psi\rangle_{k}$ for any even parity state $|\psi\rangle_{k}$ of the pair $k \bar{k}$. In terms of these operators any mixed state of these two qubits can be written as

$$
\begin{aligned}
\rho_{k k^{\prime}} & =\rho_{k} \rho_{k^{\prime}}+\frac{1}{4} C_{\mu \nu} \sigma_{k}^{\mu} \sigma_{k^{\prime}}^{\nu}, \\
\rho_{k} & =\frac{1}{2}\left(1+r_{k \mu} \sigma_{k}^{\mu}\right),
\end{aligned}
$$

where $r_{k \mu}=\left\langle\sigma_{k}^{\mu}\right\rangle$ and $C_{\mu \nu}=\left\langle\sigma_{k}^{\mu} \sigma_{k^{\prime}}^{\nu}\right\rangle-\left\langle\sigma_{k}^{\mu}\right\rangle\left\langle\sigma_{k^{\prime}}^{\nu}\right\rangle$ is the correlation tensor of the state. This representation turns out to be convenient to evaluate the quantum discord [53].

Recall that the quantum discord $D(A \mid B)$ of a state $\rho_{A B}$ of a bipartite system of distinguishable constituents $A, B$ can be defined as the minimum difference of two quantum extensions of the conditional entropy [38 40],

$$
\begin{aligned}
D(A \mid B) & =\operatorname{Min}_{\left\{\Pi_{j}\right\}} S\left(A \mid B_{\left\{\Pi_{j}\right\}}\right)-\left[S\left(\rho_{A B}\right)-S\left(\rho_{B}\right) \mid \mathrm{C} 6\right) \\
S\left(A \mid B_{\left\{\Pi_{j}\right\}}\right) & =\sum_{j} p_{j} S\left(\rho_{A / \Pi_{j}}\right),
\end{aligned}
$$

where $\rho_{A(B)}=\operatorname{Tr}_{B(A)} \rho_{A B}$ is the reduced state of subsystem $A(B)$, the set $\left\{\Pi_{j}\right\}$ describes a local measurement on $B, p_{j}=\operatorname{Tr}\left[\rho_{A B} \Pi_{j}\right]$ is the probability of result $j$ in that measurement and $\rho_{A / \Pi_{j}}=p_{j}^{-1} \operatorname{Tr}_{B}\left[\rho_{A B} \Pi_{j}\right]$ the conditional state of $A$ after such result is obtained. Evaluating $D(A \mid B)$ then requires to find the minimum over all local measurements of the conditional entropy $S\left(A \mid B_{\left\{\Pi_{j}\right\}}\right)$.

For a two qubit state and for a projective measurement along direction $\boldsymbol{k}$ in the Bloch sphere of qubit $B$, the conditional entropy (C7) reads, explicitly,

$$
S\left(A \mid B_{\boldsymbol{k}}\right)=\sum_{\mu= \pm, \nu= \pm} p_{\nu \boldsymbol{k}} f\left(\lambda_{\nu \boldsymbol{k}}^{\mu}\right)
$$

where $f(x)=-x \log _{2} x, p_{\nu \boldsymbol{k}}=\frac{1}{2}\left(1+\nu \boldsymbol{r}_{B} \cdot \boldsymbol{k}\right)$ are the probabilities of the two possible results of such measurement and

$$
\lambda_{\nu \boldsymbol{k}}^{\mu}=\frac{1}{2}\left(1+\mu\left|\boldsymbol{r}_{A}+\nu \frac{C \boldsymbol{k}}{1+\nu \boldsymbol{r}_{B} \cdot \boldsymbol{k}}\right|\right)
$$

the eigenvalues of the ensuing conditional state $\rho_{A / \nu \boldsymbol{k}}$ of qubit $A$. It was shown in ref. 53] that in the weakly correlated regime, the measurement minimizing (C8) is determined essentially by the direction of one of the singular vectors of the correlation tensor $C$.

Our interest here is in the state (31), which in the present notation is an $X$-type state symmetric under rotations around the $z$ axis. It has marginal vectors 
$\boldsymbol{r}_{k\left(k^{\prime}\right)}$ parallel to the singular vector $z_{k\left(k^{\prime}\right)}$ of $C$, i.e., $\boldsymbol{r}_{k}=\left(0,0, r_{k z}\right)$ with $r_{k z}=2\left\langle n_{k \bar{k}}\right\rangle-1$, and a correlation tensor already diagonal in the chosen basis, with

$$
\begin{aligned}
& C_{x x}=C_{y y}=2\left\langle c_{k}^{\dagger} c_{\bar{k}}^{\dagger} c_{\bar{k}^{\prime}} c_{k^{\prime}}\right\rangle, \\
& C_{z z}=4\left(\left\langle n_{k \bar{k}} n_{k^{\prime} \bar{k}^{\prime}}\right\rangle-\left\langle n_{k \bar{k}}\right\rangle\left\langle n_{k^{\prime} \bar{k}^{\prime}}\right\rangle\right) .
\end{aligned}
$$

Therefore, the minimizing measurement in the weakly correlated limit can be a projective measurement either along $z$ or along any vector $\boldsymbol{k}$ in the $x y$ plane. Beyond weak correlation, it is easy to show that for this state the previous projective measurements are still stationary. Moreover, for reduced states obtained from the ground state of the present pairing system, we have verified that the minimum is always obtained for a measurement along any vector $k$ in the $x y$ plane, which is precisely that determined by the pairing correlations (Eq. (C9)).

We then obtain $p_{\nu \boldsymbol{k}}=\frac{1}{2}$ and $\lambda_{\nu \boldsymbol{k}}^{\mu}=\frac{1}{2}\left[1+\mu \sqrt{r_{k z}^{2}+C_{x x}^{2}}\right]$ for both $\nu= \pm$. In particular, in the strong superconducting regime, Eqs. (33)- (34) lead to $r_{k z}=0$ and $C_{x x}=\frac{\Omega}{4(\Omega-1)}$, in which case Eqs. (C6) lead to

$$
D_{k k^{\prime}} \approx \frac{1}{2}\left(1-\frac{\log _{2} 3}{2}\right)\left(3+\Omega^{-1}\right),
$$

for large $\Omega$. The discord remains then finite in this limit.
The case $N=\Omega=2$. The case of $N=2$ fermions in $\Omega=2$ twofold degenerate levels is the smallest non-trivial pairing system. The exact ground state of the Hamiltonian (21) for $G \geq 0$ becomes just $|\Psi\rangle=\left(\alpha_{k} c_{k}^{\dagger} c_{\bar{k}}^{\dagger}+\right.$ $\left.\alpha_{k^{\prime}} c_{k^{\prime}}^{\dagger} c_{\bar{k}^{\prime}}^{\dagger}\right)|0\rangle$, with $k=1, k^{\prime}=2, \alpha_{k^{\prime}}=\sqrt{\frac{\lambda \pm \varepsilon}{2 \lambda}}$ and $\lambda=\sqrt{\varepsilon^{2}+G^{2}}$, which is entangled for $G>0$ (i.e., it is not a Slater determinant nor a quasiparticle vacuum). The state (31) becomes obviously pure, with $\left\langle n_{k \bar{k}} n_{k^{\prime} \bar{k}^{\prime}}\right\rangle=\left\langle\tilde{n}_{k \bar{k}} \tilde{n}_{k^{\prime} \bar{k}^{\prime}}\right\rangle=0$ and $\left\langle n_{k \bar{k}} \tilde{n}_{k^{\prime} \bar{k}^{\prime}}\right\rangle=\left|\alpha_{k}^{2}\right|$, $\left\langle c_{k^{\prime}}^{\dagger} c_{\bar{k}^{\prime}}^{\dagger} c_{\bar{k}} c_{k}\right\rangle=\alpha_{k} \alpha_{k^{\prime}}^{*}=G /(2 \lambda)$. The concurrence (32) reduces to $C=2\left|\alpha_{k} \alpha_{k^{\prime}}\right|$, i.e.,

$$
C_{k k^{\prime}}=\frac{|G|}{\sqrt{\varepsilon^{2}+G^{2}}},
$$

approaching 1 for $G / \varepsilon \rightarrow \infty$, in agreement with the limit (35) for $\Omega=2$. The quantum discord then coincides exactly with the bipartite entanglement entropy $E_{k k^{\prime}}$, which here is just $E_{\Omega-\bar{\Omega}}$, and is exactly proportional to the one-body entropy $E(|\Psi\rangle)=h\left(\rho^{\mathrm{sp}}\right): D_{k k^{\prime}}=$ $E_{k k^{\prime}}=S\left(\rho_{k \bar{k}}\right)=S\left(\rho_{k^{\prime} \bar{k}^{\prime}}\right)=E_{\Omega-\bar{\Omega}}=E(|\Psi\rangle) / 4=$ $-\sum_{k}\left|\alpha_{k}^{2}\right| \log _{2}\left|\alpha_{k}^{2}\right|=h\left(f_{k}\right)=h\left(f_{k^{\prime}}\right)$, with $I_{k k^{\prime}}=2 E_{k k^{\prime}}$ and $f_{k}=\left|\alpha_{k}^{2}\right|, k=1,2$, the eigenvalues (two-fold degenerate) of $\rho^{\mathrm{sp}}$.
[1] M.A. Nielsen and I.L. Chuang, Quantum Computation and Quantum Information (Cambridge Univ. Press, Cambridge, UK, 2000).

[2] B. Schumacher, Phys. Rev. A 51, 2738 (1995); C.H. Bennett, H.J. Bernstein, S. Popescu and B. Schumacher, Phys. Rev. A 53, 2046 (1996).

[3] R.F. Werner, Phys. Rev. A 40, 4277 (1989);

[4] C.H. Bennett, D.P. DiVincenzo, J.A. Smolin, W.K. Wootters, Phys. Rev. A 54, 3824 (1996).

[5] L. Amico, R. Fazio, A. Osterloh, V. Vedral, Rev. Mod. Phys. 80, 517 (2008).

[6] R. Horodecki, P. Horodecki, M. Horodecki, K. Horodecki, Rev. Mod. Phys. 81, 865 (2009).

[7] J. Eisert, M. Cramer, M.B. Plenio, Rev. Mod. Phys. 82, 277 (2010).

[8] C.H. Bennett, G. Brassard, C. Crepeau, R. Jozsa, A. Peres, W.K. Wootters, Phys. Rev. Lett. 70, 1895 (1993).

[9] R. Jozsa and N. Linden, Proc. R. Soc. London, Ser. A 459, 2011 (2003); G. Vidal, Phys. Rev. Lett. 91, 147902 (2003).

[10] J. Schliemann, J.I. Cirac, M. Kus, M. Lewenstein, D. Loss, Phys. Rev. A 64, 022303 (2001).

[11] J. Schliemann, D. Loss, and A.H. MacDonald, Phys. Rev. B 63, 085311 (2001).

[12] K. Eckert, J. Schliemann, D. Bruß and M. Lewenstein, Ann. Phys. 299, 88 (2002).

[13] H.M. Wiseman and J.A. Vaccaro, Phys. Rev. Lett. 91, 097902 (2003).

[14] G.C. Ghirardi and L. Marinatto Phys. Rev. A 70, 012109 (2004).

[15] C.V. Kraus, M.M. Wolf, J.I. Cirac, G. Giedke, Phys. Rev. A 79, 012306 (2009).
[16] P. Zanardi, Phys. Rev. A 65, 042101 (2002).

[17] Y. Shi, Phys. Rev. A 67, 024301 (2003).

[18] N. Friis, A.R. Lee, and D.E. Bruschi, Phys. Rev. A 87, 022338 (2013).

[19] F. Benatti, R. Floreanini, U. Marzolino, Phys. Rev. A 89, 032326 (2014).

[20] X.M. Puspus, K.H. Villegas, F.N.C. Paraan, Phys. Rev. B 90, 155123 (2014).

[21] A.R. Plastino, D. Manzano, and J. Dehesa, Europhys. Lett. 86, 20005 (2009).

[22] F. Iemini, R.O. Vianna, Phys. Rev. A 87, 022327 (2013); F. Iemini, T.O. Maciel, R. O. Vianna, Phys. Rev. B 92, 075423 (2015); T. Debarba, R.O. Vianna, F. Iemini, Phys. Rev. A 95, 022325 (2017).

[23] M. Oszmaniec, J. Gutt, M. Kuś, Phys. Rev. A 90, 020302(R) (2014).

[24] M. Oszmaniec, M. Kuś, Phys. Rev. A 90, 010302(R) (2014); Phys. Rev. A 88, 052328 (2013).

[25] G. Sárosi, P. Lévay, J. Phys. A 47, 115304 (2014); Phys. Rev. A 90, 052303 (2014); Phys. Rev. A 89, 042310 (2014).

[26] F. Iemini, T. Debarba, R.O. Vianna, Phys. Rev. A 89, 032324 (2014).

[27] N. Gigena, R. Rossignoli, Phys. Rev. A 92042326 (20fs15)

[28] A.P.Majtey, P.A.Bouvrie, A.Valdés-Hernández, A.R.Plastino, Phys. Rev. A 93, 032335 (2016).

[29] N. Gigena, R. Rossignoli, Phys. Rev. A 94042315 (2016).

[30] D. Dasenbrook, J. Bowles, J. Bohr Brask, P.P. Hofer, C. Flindt, N. Brunner, New J. Phys. 18, 043036 (2016).

[31] N. Gigena, R. Rossignoli, Phys. Rev. A 95062320 (2017).

[32] G.G. Amosov, S.N. Filippov, Quantum. Inf. Process. 16, 
2 (2017).

[33] N. Killoran, M. Cramer, M.B. Plenio, Phys. Rev. Lett. 112, 150501 (2014)

[34] N. Friis, New J. Phys. 18, 033014 (2016).

[35] C. Dunning, J. Links, H-Q. Zhou, Phys. Rev. Lett. 94 227002 (2005).

[36] S. Oh, J. Kim, Phys. Rev. B 71, 144523 (2005).

[37] C.K. Chung, C.K. Law, Phys. Rev. A 78, 034302 (2008).

[38] H. Ollivier, W.H. Zurek, Phys. Rev. Lett. 88, 017901 (2001); W.H. Zurek, Phys. Rev. A 67, 012320 (2003).

[39] L. Henderson, V. Vedral, J. Phys. A 34, 6899 (2001); V. Vedral, Phys. Rev. Lett. 90, 050401 (2003).

[40] K. Modi et al, Rev. Mod. Phys. 84, 1655 (2012).

[41] G. Adesso, T.R. Bromley, M. Cianciaruso, J. Phys. A 49, 473001 (2016).

[42] L. Ciliberti, R. Rossignoli, N. Canosa, Phys. Rev. A 82, 042316 (2010).

[43] A. Wehrl, Rev. Mod. Phys. 50, 221 (1978).
[44] V. Vedral, Rev. Mod. Phys. 74, 197 (2002).

[45] P. Ring, P. Schuck, The Nuclear Many-Body Problem (Springer, Berlin, Germany, 1980).

[46] S. Hill and W.K. Wootters, Phys. Rev. Lett. 78, 5022 (1997); W.K. Wootters, Phys. Rev. Lett. 80, 2245 (1998).

[47] H.F. Song, S. Rachel, C. Flindt, I. Klich, N. Laflorencie, K. Le Hur, Phys. Rev. B 85, 035409 (2012).

[48] J.M. Matera, R. Rossignoli, N. Canosa, Phys. Rev. A 78, 012316 (2008).

[49] V. Coffman, J. Kundu, W.K. Wootters, Phys. Rev. A 61, 052306 (2000).

[50] T.J. Osborne, F. Verstraete, Phys. Rev. Lett. 96, 220503 (2006).

[51] J. Bardeen, L.N. Cooper, J.R. Schrieffer, Phys. Rev. 108, 1175 (1957).

[52] J.A. Sheikh, P. Ring, E. Lopes, R. Rossignoli, Phys. Rev. C 66, 044318 (2002).

[53] N. Gigena, R. Rossignoli, Phys. Rev. A 90, 042318 (2014). 